09,12

\title{
Эффект кулоновской ямы в квантовых ямах (In, Ga)As/GaAs
}

\author{
(C) Р.П. Сейсян ${ }^{1}$, А.В. Кавокин ${ }^{2,3,4}$, Kh. Moumanis ${ }^{5}$, M.Э. Сасин ${ }^{1}$ \\ ${ }^{1}$ Физико-технический институт им. А.Ф. Иофрфе РАН, \\ Санкт-Петербург, Россия \\ ${ }^{2}$ University of Southampton, \\ Southampton, UK \\ ${ }^{3}$ CNR-SPIN, Viale del Politecnico 1, \\ I-00133, Rome, Italy \\ ${ }^{4}$ Санкт-Петербургский государственный университет, \\ Санкт-Петербург, Россия \\ ${ }^{5}$ Université de Sherbrooke, \\ Sherbrooke, Canada \\ E-mail: rseis@ffm.ioffe.ru \\ (Поступила в Редакцию 6 июня 2016 г. \\ В окончательной редакции 15 ноября 2016 г.)
}

\begin{abstract}
Выполнено магнитооптическое исследование экситонных переходов в высококачественных квантовых ямах гетеросистемы (In, Ga)As/GaAs. Исследование пропускания отделенных от подложки свободных образцов в магнитных полях вплоть до $12 \mathrm{~T}$ выявило богатую тонкую структуру, связанную с различными тяжелодырочными и легкодырочными экситонными переходами. В частности, были зарегистрированы переходы из возбужденных состояний легких дырок, локализованных в кулоновском потенциале, создаваемом электроном вдоль оси гетероструктуры (кулоновская яма). Последовательно принимая во внимание напряжения, образование уровней Ландау, энергии связи экситонов (диамагнитных экситонов) и эффект „кулоновской ямы“, мы смогли описать экспериментальные результаты в рамках самосогласованной вариационной процедуры. В результате были объяснены новые особенности структуры оптических переходов и с хорошей точностью были определены приведенные массы электронов и дырок для экситона, образованного как на тяжелой дырке, так и на легкой.
\end{abstract}

Работа выполнена при поддержке РФФИ и Немецкого научно-исследовательского общества (проект РФФИ-ННИО № 15-52-12018).

DOI: 10.21883 /FTT.2017.06.44486.232

\section{1. Введение}

Низкоразмерные структуры гетеросистемы (In, Ga)As/ GaAs привлекали в последние два десятилетия повышенное внимание [1-4]. И это было вызвано не только разнообразием возможных применений, но и некоторыми специфическими особенностями электронного спектра этих систем, что делает напряженные квантовые ямы (QWs) гетеросистемы (In, Ga)As/GaAs весьма многообещающими и для фундаментальных исследований. Прежде всего, практически во всем диапазоне существования стабильных квазибинарных твердых растворов, т.е. для концентраций In в пределах $x=0-0.25$, эти QWs принадлежат к смешанному типу гетеропереходов I-II-типа, так что QW представляет собой притягивающий потенциал для электрона и тяжелой дырки и отталкивающий потенциал для легкой дырки [5-9]. В общем случае предполагается, что переходы из состояний тяжелой дырки приводят к образованию пространственно-прямых экситонов, тогда как природа экситонных переходов легкой дырки не очевидна. Экситоны являются пространственнонепрямыми в QWs в четко выраженных гетеропереходах II-типа, что делает их силу осциллятора много меньше, чем в QWs гетеропереходов I-типа. Однако оптические спектры (In, Ga)As/GaAs QWs на практике показывают ярко выраженные переходы экситонов с легкой дыркой, что противоречит модели пространственно непрямых экситонов [9]. Модель кулоновской ямы (CW) позволяет разрешить это противоречие, показывая, что экситоны с легкой дыркой становятся также прямыми благодаря связывающему электрон и дырку кулоновскому взаимодействию. Эта модель была впервые предложена в [10], затем обобщена и применена к различным гетеросистемам в серии работ [11-13]. В них относительное движение электрона и дырки интерпретировалось в адиабатическом приближении, т.е. с разделением движения в плоскости ямы, и в нормальном к плоскости направлении в кинетических гамильтонианах. В таком случае одно-частичная волновая функция в направлении роста находится под действием не только прямоугольного потенциала квантовой ямы, но также и кулоновского потенциала, усредненного на волновых функциях электрона и дырки в плоскости их относительного движения. На этот последний потенциал мы и будем далее ссылаться, как на потенциал CW. Дальнейшие приближения сведутся к полному пренебрежению $\mathrm{CW}$, и разделению, таким образом, экситонного уравнения Шредингера на три независимых дифференциальных уравнения, описывающих относительное движение электрона и дырки в плоскости ямы и одномерное движе- 
ние электрона и дырки в перпендикулярном плоскости ямы направлении. Такое разделение общего уравнения Шредингера невозможно для широкого класса систем, в которых эффекты кулоновской ямы более существенны. В качестве примера можно привести $\mathrm{CdS}$ и $\mathrm{CdSe}$ микрокристаллы $[14,15]$. Параболическое приближение $\mathrm{CW}$, принятое в [10], привело к хорошему согласию с экспериментальными данными по тонкой структуре экситонного спектра в 0-мерных микрокристаллах $\mathrm{CdS}$ и $\mathrm{CdSe}$, помещенных в стеклообразную матрицу $[14,15]$, где вполне приемлемо приближение бесконечной высоты барьера, соответствующего границе микрокристалла.

B обычных квантовых ямах I-типа CW-эффекты оказываются не очень существенными, так как потенциал квантовой ямы в этом случае вносит доминирующий вклад в ограничение движения носителей заряда. Однако в гетеросистемах типа II (т.е. когда яма для электронов образует барьер для дырок, или наоборот), или в системах с переходом тип I-тип II, CW-эффект играет ключевую роль. Это было продемонстрировано в серии статей по квантовым ямам CdTe/CdMnTe и $\mathrm{GaAs} / \mathrm{InGaAs}$, в которых CW-потенциал оказывает решающее влияние и на спектр энергетических состояний, и на силы осциллятора переходов [11-13].

В настоящей работе мы рассматриваем воздействие CW на тонкую структуру экситонных переходов тяжелых и легких дырок в высококачественных квантовых ямах гетеросистемы (In, Ga)As/GaAs, помещенной в магнитное поле. Магнитное поле оказывает сильное влияние на электрон-дырку в плоскости их относительного движения и на эффективный $\mathrm{CW}$-потенциал, что приводит к изменениям энергии связи экситона и к существенному перераспределению силы осциллятора между основным и возбужденными состояниями экситона. Экспериментальные данные по воздействию $\mathrm{CW}$-эффектов на экситонные спектры гетероструктур (In, Ga)As/GaAs с квантовыми ямами впервые были получены при изучении тонкой осциллирующей структуры экситонных переходов - диамагнитных экситонов (DE) $[8,9]$. В настоящей работе мы сообщаем о наблюдении уникальной, очень богатой тонкой структуры экситонных переходов в этих объектах, которая, в основном, объясняется в рамках нашей теоретической модели, но частично все еще остается без удовлетворительного толкования. Статья организована следующим образом. В разд. 2, следующей после данного введения, мы представим используемый формализм, позволяющий описывать тонкую структуру экситонных спектров с учетом $\mathrm{CW}$-эффекта. В разд. 3 описываются образцы и техника эксперимента. В разд. 4 приводятся наиболее типичные экспериментальные спектры поглощения, полученные на наших образцах. В разд. 5 мы приводим анализ экспериментальных данных. Сравнивая их с результатами вычислений, мы предлагаем интерпретацию наблюдаемой тонкой структуры экситонных переходов. В разд. 6 мы обсуждаем особенности эффекта кулоновской ямы. И наконец, в разд. 7 мы предложим общее заключение.

\section{2. Теоретические основы}

2.1. Зонная схема квантовых ям в гетероструктурах (In, Ga)As/GaAs. Рассмотрим сначала энергии в деформированных квантовых ямах гетероструктуры (In, Ga)As/GaAs в одночастичном приближении. Тонкие слои $\operatorname{In}_{x} \mathrm{Ga}_{1-x}$ As имеют решетку, не вполне совпадающую с решеткой подложки GaAs, что приводит к увеличению ширины запрещенной зоны и к расщеплению между уровнями тяжелых и легких дырок, притом потенциальная яма становится глубже для тяжелых дырок и обращается в нуль для легких дырок. Потенциал легких дырок как функция содержания индия $x$ может быть записан следующим образом:

$$
E_{l h}=\Delta E_{v}(x)+\Delta E_{v}^{H}(e)-\frac{\Delta e}{2}-\frac{9}{16} \frac{\Delta_{e}^{2}}{\Delta_{0}(x)},
$$

где $\Delta E_{v}(x)$ - разность между энергиями вершин валентных зон недеформированных $\operatorname{In}_{x} \mathrm{Ga}_{1-x} \mathrm{As}$ и $\mathrm{GaAs}, \Delta E_{v}^{H}(e)$ является краем валентной зоны, перенормированным из-за гидростатической деформации при относительной деформации $e=-x /(13.9+x)$, $\Delta_{e}$ - деформационное расщепление валентной зоны, $\left.\Delta_{e}=\left|b_{x}\right|\left(1+\lambda_{x}^{100}\right) e\right) ;$ согласно $[16,17] b_{x}=-1.7-0.1 x$ и $\Delta_{0}(x)=0.341-0.09 x+0.40575 x^{2}$. Здесь все анизотропные константы приведены для плоскости (100) кристалла и энергии даны в eV.

Предполагая, что отношение гидростатических констант деформации для зоны проводимости и валентной зоны не зависит от содержания индия, можно переписать уравнение (1) следующим образом:

$$
\begin{aligned}
E_{l h}= & \Delta E_{v}(x) \frac{a^{*}\left(2-\lambda_{x}^{100}\right)}{1+Q_{e}^{H}} \frac{x}{13.9+x}-\frac{\left|b_{x}\right|}{2}\left(1+\lambda_{x}^{100}\right) \\
& \times \frac{x}{13.9+x}\left[1+\frac{9}{16} \frac{\left|b_{x}\right|}{2 \Delta_{0}(x)}\left(1+\lambda_{x}^{100}\right) \frac{x}{13.9+x}\right],
\end{aligned}
$$

где $a^{*}=-8.68+2.77 x-$ постоянная гидростатической деформации.

Здесь, следуя [17], можно подставить

$$
a^{*} /\left(1+Q_{e}^{H}\right)=1.16-0.16 x .
$$

В качестве альтернативы можно непосредственно ввести в качестве $Q_{e}^{H}=0.89 / 0.11$. Все остальные параметры хорошо известны [18]. Отметим, что Leymarie и др. [7] сообщили другое значение отношения смещения зон в (In, Ga)As/GaAs. Но, для большей последовательности, мы будем использовать далее значение, полученное из уравнения (3), а не экспериментальное - из [7].

Описываемый подход позволяет получить зависимость энергии легких дырок от концентрации индия в $\operatorname{In}_{x} \mathrm{Ga}_{1-x} \mathrm{As}$, которая показана на рис. 1. Кроме того показаны потенциалы тяжелых дырок и электронов, рассчитанные таким же образом, а также уровни энергии 


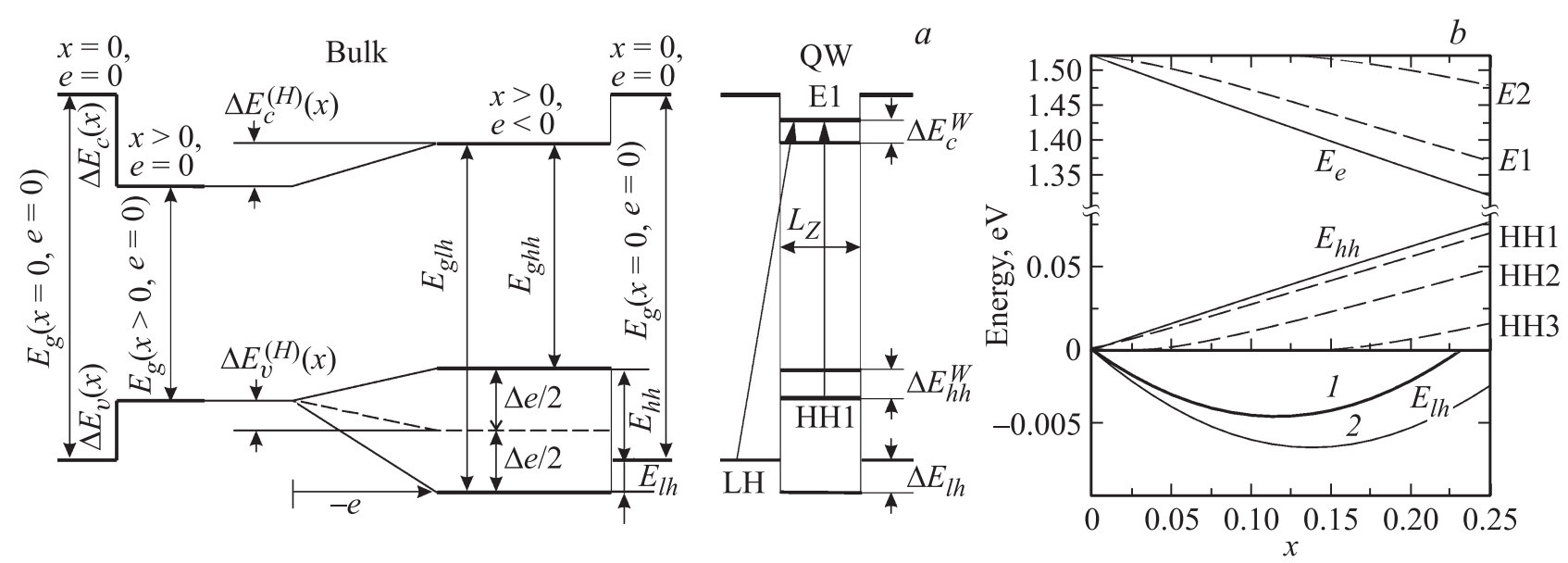

Рис. 1. Зонная схема деформированной квантовой ямы в гетероструктуре (In, Ga)As/GaAs (a). Стрелки показывают оптически разрешенные межзонные переходы. Изменение зонных энергий электронов, тяжелых и легких дырок в деформированном слое $\operatorname{In}_{x} \mathrm{Ga}_{1-x} \mathrm{As}(b)$ показано как функция концентрации индия $x .1$ и 2 - две различные аппроксимации разрыва зон, обсуждаемые в тексте.

электронов и тяжелых дырок в квантовой яме толщиной $8 \mathrm{~nm}$. Можно видеть, что в соответствии с моделью при $x=0-0.25$ потенциал квантовой ямы для легкой дырки оказывается второго типа, притом для легких дырок разрыв зон оказывается самым большим в области $x=0.13-0.16$. Максимальная величина разрыва зон составляет около $6 \mathrm{meV}$. Коррекция перенормировки при учете разницы эффективных масс легких дырок в GaAs и $\mathrm{In}_{x} \mathrm{Ga}_{1-x}$ As для этого диапазона $x$ и для экситонного боровского радиуса $10 \mathrm{~nm}$ составляет около $2 \mathrm{meV} \mathrm{[12],} \mathrm{так}$ что в итоге мы получаем для легкой дырки потенциал типа II с максимальной высотой барьера $8 \pm 3 \mathrm{meV}$. Эти результаты согласуются с выводами Leymarie и др. [7].

2.2. Эффект кулоновской ямы и его описание. Попробуем теоретически описать экситон, заключенный в квантовую яму с почти нулевым разрывом валентной зоны в присутствии магнитного поля, перпендикуляного к плоскости квантовой ямы. Разделив движение экситонного центра масс и относительное движение электрона и дырки в плоскости квантовой ямы, как в [19], и полагая, что экситон не перемещается как целое в плоскости QW, мы получаем следующий экситонный гамильтониан:

$$
\hat{H}=\hat{H}_{e}+\hat{H}_{h}+\hat{H}_{\mathrm{ex}},
$$

где

$$
\begin{gathered}
\hat{H}_{e}=-\frac{\hbar^{2}}{2 m_{v}} \frac{\partial^{2}}{\partial z_{v}^{2}}+V_{v}\left(z_{v}\right)-\mu_{B} g_{v} \mathbf{s}_{v} \mathbf{B}+(l+1 / 2) \hbar \omega_{c}^{v}, \\
v=e, h \\
\hat{H}_{\mathrm{ex}}=-\frac{\hbar^{2}}{2 \mu}\left[\frac{1}{\rho} \frac{\partial}{\partial \rho}\left(\rho \frac{\partial}{\partial \rho}\right)-\frac{\rho^{2}}{4 L^{4}}\right]-\frac{e^{2}}{\varepsilon \sqrt{\rho^{2}+\left(z_{e}-z_{h}\right)^{2}}} .
\end{gathered}
$$

Здесь магнитная длина $L=\sqrt{\frac{c \hbar}{e B}}, \mathbf{B}-$ магнитное поле, $m_{e(h)}$ - масса электрона (дырки) в плоскости, перпендикулярной к плоскости ямы, $V_{e(h)}$ - потенциал квантовой ямы для электрона (дырки), $g_{e(h)}$ и $\omega_{c}^{e(h)}-g$-факторы электрона (дырки) и циклотронные частоты соответственно, $l=0,1,2, \ldots, \mu=m_{e} m_{h}^{\|}\left(m_{e}+m_{h}^{\|}\right)^{-1}, m_{h}^{\|}-$ дырочная масса в плоскости ямы, $\varepsilon$ - диэлектрическая проницаемость. Здесь и далее мы пренебрегаем смешиванием легкой и тяжелой дырок.

Подобные экситонные задачи были рассмотрены в ряде работ [20,21]. В [20] выполнен вариационный расчет экситонных параметров при наличии магнитного поля, в квантовой яме типа I, в [21] и в работах, там упомянутых; вариационный расчет основного состояния экситона в QW с переходом тип I-II дан в [22,23], там же был сформулирован подход, позволяющий выполнить расчет возбужденных экситонных состояний в QW вблизи перехода тип I-тип II. В настоящей работе мы скомбинируем подходы $[8,9,22,23]$, чтобы вычислить энергии связи и радиационное затухание основного и возбужденных экситонных состояний в QW с переходом тип I-тип II в присутствии внешнего магнитного поля. Пробная функция выбрана в виде

$$
\Psi_{\mathrm{exc}}\left(z_{e}, z_{h}, \rho\right)=U_{e}\left(z_{e}\right) U_{h}\left(z_{h}\right) f(\rho),
$$

где $z_{e}, z_{h}$ - координаты электрона (дырки) по нормали к плоскости ямы, $\rho-$ координата электроннодырочных пар в плоскости их относительного движения. Поскольку разрыв зоны проводимости в нашей системе велик, мы находим $U_{e}\left(z_{e}\right)$ как решение одночастичной вариационной задачи для прямоугольной QW. Функции $U_{h}\left(z_{h}\right)$ и $f(\rho)$ определяются системой двух интегродифференциальных уравнений

$$
\begin{gathered}
\iint d z_{e} d z_{h} U_{e}\left(z_{e}\right) U_{h}\left(z_{h}\right)(\hat{H}-E) \Psi_{\mathrm{exc}}=0, \\
\iint 2 \pi \rho d \rho d z_{e} U_{e}\left(z_{e}\right) f(\rho)(\hat{H}-E) \Psi_{\mathrm{exc}}=0 .
\end{gathered}
$$


Если кулоновским членом в уравнениях $(6,6 \mathrm{a})$ пренебрегают, то $f(\rho) \rightarrow(\sqrt{\pi R})^{-1}$, где $R$ есть радиус образца. Уравнение (6а) дает в этом случае $E_{e h}-$ энергию электроннодырочной пары. Определим далее энергию связи экситона $R^{*}$ как

$$
R^{*} \equiv E_{e h}-E
$$

где $E$ - собственная энергия системы $(6,6 \mathrm{a})$ с учетом кулоновского притяжения электрон-дырка. Заметим, что в квантовой яме с почти нулевым разрывом валентной зоны движение дырки в направлении $z$ ограничено в основном за счет кулоновского потенциала $[8,9]$, создаваемого электроном (см. рис. 2)

$$
V_{h}\left(z_{h}\right)=-\frac{e^{2}}{\varepsilon} \iint 2 \pi \rho d \rho d z_{e} \frac{U_{e^{2}}\left(z_{e}\right) f^{2}(\rho)}{\sqrt{\rho^{2}+\left(z_{e}-z_{h}\right)^{2}}} .
$$

В непосредственной близости к точке перехода тип I-тип II экситонные состояния с более высокими дырочными квантовыми числами (2,3 и т.д.) могут дать значительные вклады в спектры. Для расчета волновых функций и энергий связи этих более высоких экситонных состояний мы будем аппроксимировать потенциал (8) следующим образом:

$$
V_{h}\left(z_{h}\right) \approx-\frac{V_{0}}{\cosh ^{2}\left(\alpha z_{h}\right)},
$$

где $V_{0}$ и $\alpha-$ это подгоночные параметры, дающие наилучшее соответствие с (8). В этом потенциале волновые функции дырок можно найти аналитически. Слабый потенциал CW затем учитывается в первом порядке

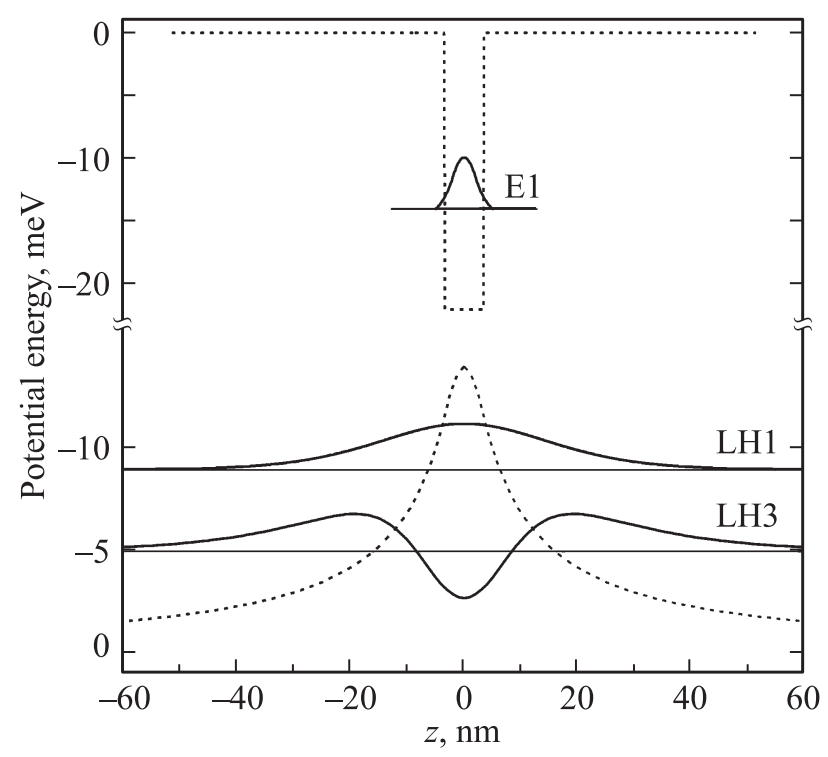

Pис. 2. Огибающие функции электронов и легких дырок для квантовой ямы толщиной $7 \mathrm{~nm}$ в гетероструктуре $\mathrm{In}_{0.06} \mathrm{Ga}_{0.94} \mathrm{As} / \mathrm{GaAs}$. Волновые функции легких дырок показаны для основного (LH1) и третьего (LH3) состояний. Также показаны потенциальные ямы зоны проводимости и кулоновский потенциал ямы (штриховые линии). теории возмущений (подробнее см. [23]). Знание $U_{h}\left(z_{h}\right)$ позволяет из (6а) получить функцию относительного электрон-дырочного движения как решение однопараметрической вариационной задачи.

В качестве пробной функции мы используем

$$
f(\rho)=\sqrt{\frac{2}{\pi}} \frac{1}{a} \exp (-\rho / a),
$$

где $a-$ вариационный параметр. Самосогласованное решение уравнений $(6,6 a)$ затем получается после ряда итераций. Таким способом мы определяем $g$-факторы электронов и дырок в нулевом приближении как

$$
g_{v}=g_{W}^{v} \theta_{W}+g_{B}^{v} \theta_{B}^{v},
$$

где $\theta_{W}^{v}=\int_{-L_{z} / 2}^{L_{z} / 2} d z U_{v}^{2}(z), \theta_{B}^{v}=1-\theta_{W}^{v}=1-\theta_{W}^{h}$, а $g_{W}^{v}$ и $g_{B}^{v}$ являются $g$-факторами материала ямы и барьерных материалов соответственно, $v=e, h, L_{z}$ - ширина квантовой ямы.

Заметим, что описанная выше процедура самосогласованного вариационного расчета, строго говоря, действительна только для основного состояния экситона. Если применять аналогичную процедуру для нахождения экситонного состояния, связанного с одним из верхних уровней дырки в условиях потенциала кулоновской ямы (9), придется пересчитывать точный потенциал (8) и приближенный потенциал (9). Волновые функции возбужденных состояний, полученные таким образом, не будут ортогональными к основному состоянию, что приведет к противоречию с основными принципами квантовой механики. Для того чтобы избежать этого противоречия, в расчетах возбужденных состояний дырок мы используем тот же CW-потенциал, который найден для основного состояния. Это позволяет сохранить ортогональность, но, к сожалению, снижает точность вариационного расчета для возбужденных экситонных состояний. В частности, он приводит к одному и тому же значению боровского радиуса для всех экситонных переходов E1LHN $(N=1,2,3, \ldots)$.

Отмеченное ограничение справедливости самосогласованного вариационного подхода к решению уравнения Шредингера для экситона в CW является фундаментальным, поскольку обусловлено ограниченной точностью адиабатического приближения, которое является одним из краеугольных камней рассматриваемой модели. Отметим также, что для определения энергий экситонных переходов E1LHN наша модель тем не менее работает хорошо и обеспечивает, как будет показано ниже, замечательное согласие с экспериментальными данными.

Радиационное затухание экситона можно выразить с помощью полученных экситонных характеристик как

$$
\hbar \Gamma_{0}=\hbar \omega_{0} \omega_{L T} \sqrt{\varepsilon} a_{B}^{3} a^{-2} J_{e h}^{2} .
$$

$J_{e h}=\int d z U_{e}(z) U_{h}(z), \omega_{0}$ здесь резонансная частота экситона, $\omega_{L T}$ - продольно-поперечное расщепление и $a_{B}-$ боровский радиус объемного экситона. (Для GaAs можно использовать $\omega_{L T}=0.08 \mathrm{meV}$ и $\left.a_{B}=14 \mathrm{~nm}\right)$. 
2.3. Смешивание тяжелых и легких дырок. Обычно, в приближении эффективной массы, состояния дырок в алмазоподобных кристаллах описываются гамильтонианом Латтинжера, и вблизи $\mathbf{k}=0$ дисперсия носителей заряда определяется так называемыми параметрами Латтинжера: $\gamma_{1}, \gamma_{2}, \gamma_{3}$, зависящими от состава, ориентации кристалла и гофрировки валентной зоны. В случае квантовой ямы - в рамках теории возмущений - вырождение валентной зоны снимается, и эффективные массы на соответствующих квантовых уровнях задаются как $m_{0} / m_{v}=\gamma_{1} \mp \gamma($ где $(+)$ теперь соответствует уровням тяжелой дырки и $(-)$ - уровням легкой дырки. Таким образом, бывшие легкие дырки приобретают относительно тяжелые массы в плоскости ямы, в то время как тяжелые дырки становятся легче из-за сильного взаимодействия между этими состояниями. Приближенная формула для эффективных масс, приведенная выше, не принимает во внимание возможность туннелирования легких дырок в барьерные слои и влияние напряжений на расщепление легкой и тяжелой дырок в материале ямы. Эти обстоятельства становятся важными в случае гетероструктур (In, Ga)As/GaAs, напряженных из-за несоответствия постоянных решетки в материалах ямы и барьера, что приводит к возникновению LH1-HH1 расщепления, увеличивающегося с ростом содержания индия. Такая модель была теоретически исследована в [24], где было показано, что дырочные массы в квантовой яме сильно зависят от одноосной составляющей напряжения и от туннелирования легких дырок, а также от ориентации кристалла. Для простоты мы рассмотрим только одну кристаллографическую ориентацию слоя QW, которая соответствует нашим образцам, выращенным на поверхностях, ориентированных по (100). Следуя [24], мы найдем безразмерные обратные массы $\left(M v=m_{0} / m_{v}\right)$, которые могут быть записаны в виде

$$
M_{v N p}=M_{v}\left(1+C_{v V} \Phi_{p}^{*}\right)+\Delta M_{v V},
$$

где $M_{v}=\gamma_{1} \pm 2 \gamma_{2}-$ обратные массы дырок в объемном материале, $\quad C_{v N}=3 \gamma_{3}^{2}\left(M_{v} \mp 4 \varepsilon_{100}^{(N)}\right) /\left(M_{v}\left(\gamma_{2}-\varepsilon_{100}^{(N)}\right)^{2}\right.$, $\Delta M_{v V}= \pm 3\left(\gamma_{3}^{2}-\gamma_{2}^{2}+\varepsilon_{100}^{N}\right) /\left(\gamma_{2}-\varepsilon_{100}^{(N)}\right), \varepsilon_{100}^{(N)}=\bar{b} e_{100} / 2 N^{2}$, $\bar{b} e_{100}=(b / R y)\left(L_{z} / \pi a_{0}\right)^{2} e(x)\left(\left(C_{11}+2 C_{12}\right) / C_{11}\right), \quad R y=$ $=13.6 \mathrm{eV}, a_{0}=0.53 \AA-$ боровский радиус, а $C_{11}, C_{12}$ являются модулями упругости пленки.

$$
M_{v N p}=M_{v}\left(1+C_{v N} \Phi_{p}^{*}\right)+\Delta M_{v N} .
$$

В случае существенного вклада туннелирования фазовый множитель $\Phi_{p}^{*}$ может быть записан как

$$
\begin{aligned}
\Phi_{p}^{*}= & \Delta E_{v}^{2} /\left(E_{v N}+\Delta E_{v}\right)\left[E_{v N}\left(1-M_{v}^{(W)} / M_{v}^{(B)}\right)\right] \\
& \times\left(k_{l h} L_{z} Z_{l h,-p}\left(E_{v N}\right)\right)
\end{aligned}
$$

где $\quad Z_{l h,-p}\left(E_{v N}\right)=\left(M_{l h}^{(W)} k_{l h} / M_{l h}^{(B)} \chi_{l h}\right)-p \operatorname{ctg}^{p}\left(k_{l h} L_{z} / 2\right)$, $p$ - четность уровня $N, \quad p=(-1)^{N+1}, \quad k_{l h}=$ $=\sqrt{2 m_{0}\left(-E+\bar{b} e_{100}\right) / \hbar^{2} M_{l h}^{(W)}}-$ волновой вектор лег- кой дырки в яме и $\chi_{l h}=\sqrt{2 m_{0}\left(-E+\Delta E_{v}\right) / \hbar^{2} M_{l h}^{(B)}}-$ абсолютная величина волнового вектора легкой дырки в барьере, $M_{v}^{(W)}, M_{v}^{(B)}$ - безразмерные обратные массы в слоях ямы и барьера, $E$ - энергия, измеренная от вершины валентной зоны материала ямы, $k_{l h}$ и $Z_{l h,-p}$ должны вычисляться для энергии $E=E_{h h N}$.

Длина туннелирования легких дырок обычно сравнима с шириной ямы $a$ из-за малой величины отношения масс дырок $\beta(n) \ll 1$. Как следует из уравнения (14), туннелирование легких дырок может сильно повлиять на эффективную массу тяжелых дырок. Это верно, если $m_{l h} \Delta E_{v} L_{z}^{2} / \pi^{2} \hbar^{2} \leq 1\left(\chi_{l h} L_{z} \leq 1\right)$. Усиление проникновения волновой функции легких дырок в барьеры с уменьшением ширины ямы эффективно уменьшает вклад легких дырок в дисперсию подзоны НH1. Coответственно масса $m_{h h l}$ увеличивается монотонно до значений порядка $\sqrt{m_{l h} / m_{h h}}$ при $L_{z} \approx \hbar / \sqrt{m_{h h}} \Delta E_{v}$.

Как показано в разд. 2.1, расщепление между первыми уровнями легких и тяжелых дырок в квантовых ямах (In, Ga)As/GaAs возрастает с увеличением $x$, что приводит к переходу тип I-тип II для легкой дырки. Это можно рассматривать как предельный случай модели, разработанной в [23]. Можно ожидать при этом резкое увеличение массы тяжелых дырок относительно значения, предсказанного теорией Латтинжера для чистого 2D-случая.

\section{3. Образцы и техника эксперимента}

Образцы $\operatorname{In}_{x} \mathrm{Ga}_{1-x}$ As были выращены методом молекулярно-пучковой эпитаксии на (100) ориентированных подложках GaAs. Для того, чтобы затем обеспечить полное удаление подложек путем химического травления, на GaAs предварительно выращивались $0.5 \mu \mathrm{m}$ толщиной стоп-слои $\mathrm{Al}_{0.5} \mathrm{Ga}_{0.5} \mathrm{As}$. Характеристики исследуемых образцов приведены в табл. 1. Удаление подложек позволило обнаружить тонкую структуру спектров пропускания, далее используемых для восстановления спектральной зависимости коэффициента поглощения в гетероструктурах с множественными квантовыми ямами.

Магнитооптические измерения проводились при $T=1.7 \mathrm{~K}$ в гелиевом криостате с откачкой паров гелия, со сверхпроводящим соленоидом, позволявшим создавать магнитные поля до $11 \mathrm{~T}$. Свободные образцы (без приклейки) погружались непосредственно в среду жидкого гелия. Спектры были получены для левого и правого циркулярно поляризованного света в геометрии Фарадея с помощью светосильных дифракционных монохроматоров. Измерения магнитопоглощения проводились при нормальном падении света. Более подробно наша экспериментальная методика описана в работах [25-27].

Как видно из табл. 1, низкие концентрации индия $(x=0.03-0.07)$ соответствуют лучшему качеству образцов $^{1}$ и ультраузким спектральным линиям. Чтобы

\footnotetext{
${ }^{1}$ Образцы были изотовлены в Оптическом научном центре университета Аризоны, США, в группе Х. Гиббса и Г. Хитровой.
} 
Таблица 1. Параметры исследуемых образцов

\begin{tabular}{c|c|c|c|c|c|c}
\hline $\begin{array}{c}\text { Номер } \\
\text { образца }\end{array}$ & Образец & $x$ & $\begin{array}{c}L_{Z}, \\
\mathrm{~nm}\end{array}$ & $\begin{array}{c}L_{B}, \\
\mathrm{~nm}\end{array}$ & $N$ & $\begin{array}{c}\text { FWHM, } \\
\mathrm{meV}\end{array}$ \\
\hline 1 & NMC33 & 0.033 & 9.3 & 89.7 & 30 & 0.8 \\
2 & NMC31 & 0.044 & 8.5 & 83.7 & 20 & 0.9 \\
3 & NMC21 & 0.045 & 9.0 & 82.2 & 20 & 1.1 \\
4 & NMC11 & 0.062 & 8.8 & 75.8 & 20 & 1.7 \\
5 & NMC15 & 0.066 & 6.3 & 79.4 & 20 & 1.6 \\
6 & GIBQW & 0.132 & 4.4 & 14.7 & 30 & 6.6 \\
7 & MOD22 & 0.161 & 7.5 & 33.1 & 30 & 5.0 \\
8 & 4K5 & 0.209 & 5.0 & 13.0 & 30 & 10.3 \\
9 & MOD23 & 0.225 & 4.6 & 23.7 & 30 & 11.9
\end{tabular}

Пр и м е чан и е. $x-$ концентрации индия, $L_{Z}-$ толщина слоя и $L_{B}-$ толщина барьера (эти параметры получены из измерений дифракции рентгеновских лучей), $N$ - число периодов в структурах с множественными квантовыми ямами; FWHM является полной шириной спектральных линий на полувысоте для состояний HH1E1. FWHM непосредственно взято из измеренных спектров поглощения.

исключить эффекты релаксации напряжений, барьеры в нескольких структурах QW были сделаны намного толще, чем ямы. Концентрация In, $x$, ширина ямы, $L_{Z}$, и ширины барьеров, $L_{B}$, были определены с использованием рентгеновской дифракционной спектроскопии ${ }^{2}$. Для того, чтобы выявить влияние $\mathrm{CW}$ на тонкую структуру экситонных переходов в гетеросистеме $\operatorname{In}_{x} \mathrm{Ga}_{1-x} \mathrm{As} / \mathrm{GaAs}$, были выбраны образцы с низким содержанием In, прежде всего, по двум причинам. Во-первых, как следует из рис. 1, выбранные нами образцы должны демонстрировать простейшие экситонные спектры, так как в QW шириной менее $100 \AA$ существует только одно состояние электрона; во-вторых, низкая концентрация индия обеспечивает наилучшее качество образцов и ультраузкие спектральные линии (табл. 1). Образцы с более высоким содержанием In были использованы только для сравнения.

\section{4. Экспериментальные результаты}

4.1. Оригинальные спект ры $(B=0)$. Оптические спектры пропускания образцов $\mathrm{c}=0.033-0.066$, измеренные либо до, либо после удаления подложки, показаны на рис. 3. Свободные образцы демонстрируют гораздо больше спектральных деталей. Можно отличить два вида спектров, имеющих разные масштабы модуляции поглощения. Относительно низкие амплитуды спектральных линий соответствуют экситонным переходам в слоях $\operatorname{In}_{x} \mathrm{Ga}_{1-x} \mathrm{As}$, в то время как более сильные спектральные линии соответствуют переходам в барьерных слоях GaAs. Последние характеризуются сложной много-пичковой системой переходов и могут быть описаны в терминах экситон-поляритонных ветвей квантования в сочетании со слабыми эффектами деформации [25]. В спектрах, полученных до удаления

\footnotetext{
2 Рентгеновские измерения были выполнены Н.Н. Фалеевым в ФТИ им. А.Ф. Иоффе.
}
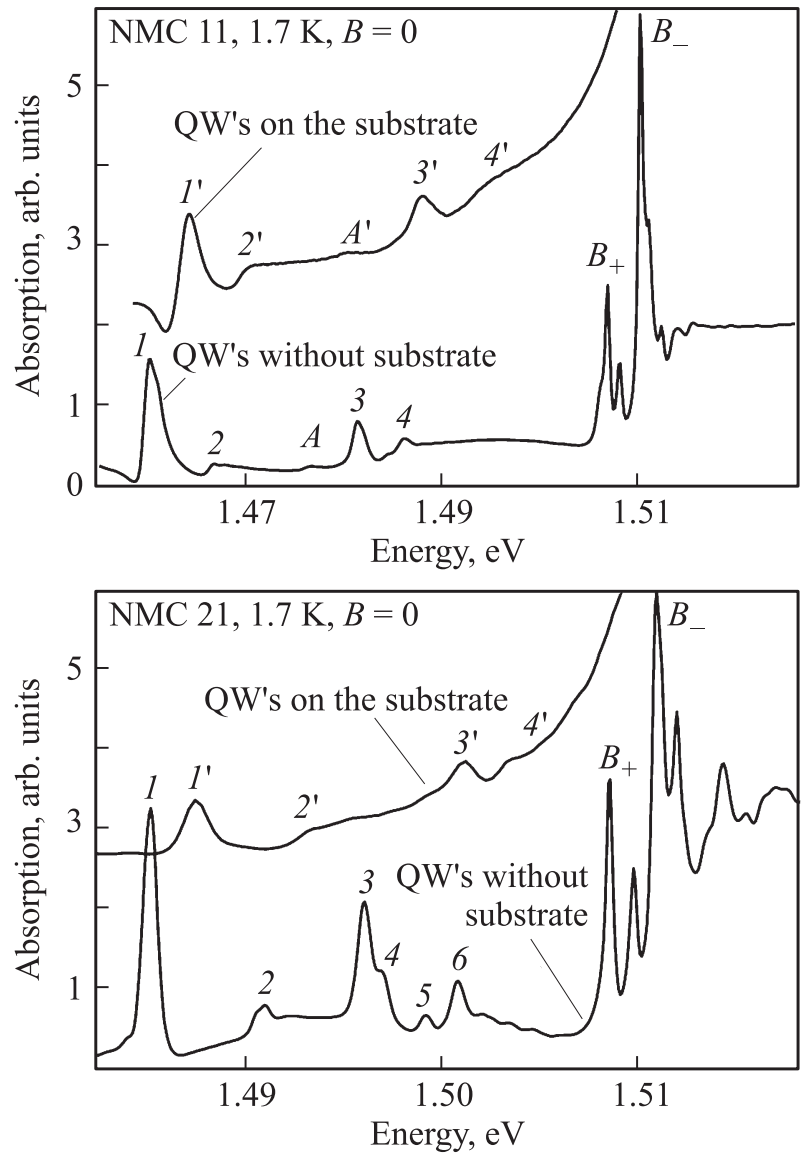

Рис. 3. Оптические спектры поглощения двух наших образцов (NMC11 и NMC21) до и после удаления подложки. $1,1^{\prime}-\mathrm{HH} 1 \mathrm{E} 1(1 s), 2,2^{\prime}-\mathrm{HH} 1 \mathrm{E} 1(2 s), 3,3^{\prime}-\mathrm{LH} 1 \mathrm{E} 1$, 6 - LH3E1, B+ и $\mathrm{B}_{-}-$-экситоны с легкими и тяжелыми дырками в барьерном слое GaAs.

подложки, многие детали теряются, естественно. В частности, утрачиваются детали поведения барьера GaAs. Все положения пиков в свободных образцах оказываются сдвинутыми в длинноволновую сторону примерно на 2.5-6.6 meV (см. табл. 2). Сдвиги $\Delta_{1}$ и $\Delta_{2}$ для экситонов на тяжелых и легких дырках соответственно отличаются.

Далее мы рассмотрим линии относительно малой амплитуды, связанные с переходами в квантовой яме. Эти линии, в свою очередь, могут быть разделены на две группы: одна - это линии с коротковолновым сател-

Таблица 2. Деформации (e), сдвиги и расщепления $(\Delta)$ в исследуемых образцах: эксперимент и расчет.

\begin{tabular}{c|c|c|c|c|c|c}
\hline Образец & $x$ & $\begin{array}{c}e, \\
10^{-4}\end{array}$ & $\begin{array}{c}\Delta_{1}, \\
\text { (теор.) }\end{array}$ & $\begin{array}{c}\Delta_{1}, \\
\text { (экс.) }\end{array}$ & $\begin{array}{c}\Delta_{2}, \\
(\text { теор.) }\end{array}$ & $\begin{array}{c}\Delta_{2}, \\
(\text { экс.) }\end{array}$ \\
\hline NMC21 & 0.045 & 4.3 & 2.1 & 2.4 & 5.1 & 5.4 \\
NMC11 & 0.062 & 5.8 & 2.9 & 3.75 & 6.9 & 6.25
\end{tabular}

Примечание. Измерения были сделаны после удаления подложки на обоих образцах. Сдвиги и расщепления даны в $\mathrm{meV}$. 
литом, связанные с НН1Е1-экситоном (1s) состояниями и с первыми возбужденными $(2 s)$ состояниями, соответственно, и ряд линий, соответствующих состояниям экситона на легкой дырке. Следует отметить, что для небольшого содержания In в системе, в соответствии с расчетной схемой, нет других разрешенных оптических переходов, следовательно, все более высокоэнергетические линии, за исключением тех, которые связаны с экситоном HН1Е1, должны быть связаны с экситонами на легких дырках.

4.2. Поведение оптических спектров в сильных магнитных полях. Серия спектров поглощения, измеренных в сильных магнитных полях, для одного из образцов показана на рис. 4. Имеется много сильных максимумов, также как и слабых. Все они испытывают голубой сдвиг, когда магнитное поле возрастает, так что количество ярко выраженных пиков ограничено из-за сильного поглощения света в барьерных слоях. Для справки показаны также спектры магнитопропускания образцов, измеренные перед удалением подложки (рис. 5). Отчетливо видно, что есть одна группа линий с очень типичным поведением для магнитоэкситона (ДЭ) в QW I типа [26,27], которые могут быть отнесены к экситону HH1E1.

Есть две дополнительные особенности, возникающие в магнитном поле, на спектрах HH1E1 у свободных образцов (см. веерные диаграммы). Во-первых, основное состояние, как выясняется, имеет слабые длинноволновые спутники, сдвинутые на $\sim 1 \mathrm{meV}$ по отношению к основной линии (рис. 6, образец 3). Такие спутники также наблюдаются на рис. 7 , где показаны веерные диаграммы образца 4. Однако они находятся на коротковолновой стороне основной спектральной линии. Второй особенностью является дополнительная система линий, которая экстраполируется при уменьшении магнитного поля до нуля, на ту же область экситонных состояний, что и основное состояние.

Однако самым интересным является поведение экситонных переходов на легких дырках. Их можно рассмотреть путем мысленного исключения всех выше рассмотренных экситонных линий HH1E1. Видно, что экситоны на легких дырках практически не образуют свою собственную веерную диаграмму и их энергии первоначально лишь слабо зависят от магнитного поля. К тому же, в магнитном поле появляется вторая линия, сдвинутая относительно основного состояния перехода аналогично первому возбужденному $(2 s)$ состоянию HH1E1-экситона. Притом понятно, что эта линия не соответствует ни одному из возбужденных состояний LH1E1 экситона. При более сильных полях две линии дублета расходятся в правой круговой поляризации (RCP) и сходятся в левой круговой поляризации $(\mathrm{LCP})$, как если у них имеются противоположные знаки $g$-фактора. Этот эффект проявляется только в свободных образцах, в то время как в образцах, имеющих подложку, зависимость положения этих резонансов от магнитного поля оказывается квазипараллельной.

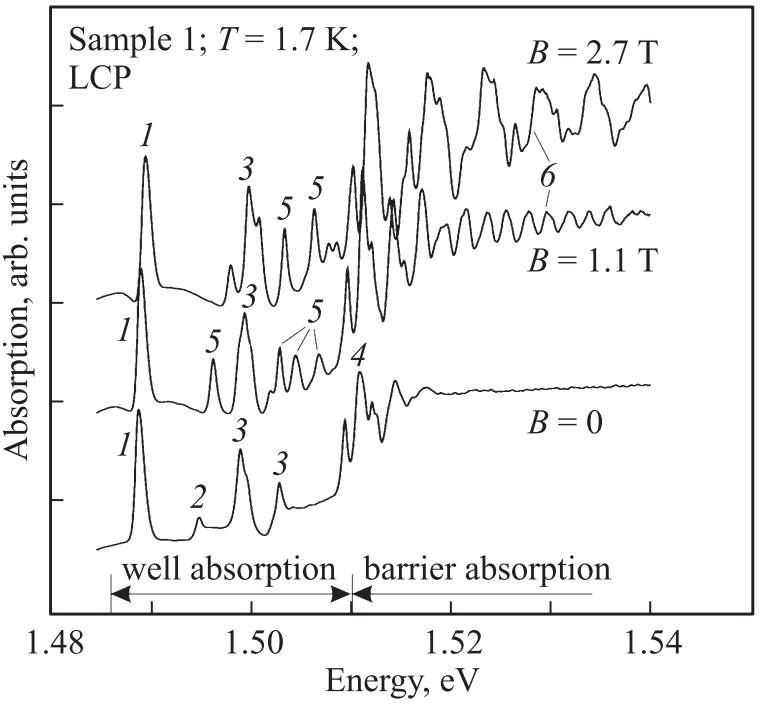

Рис. 4. Спектры поглощения образца 1 при различных магнитных полях (подложка удалена). Левая круговая поляризация (LCP).

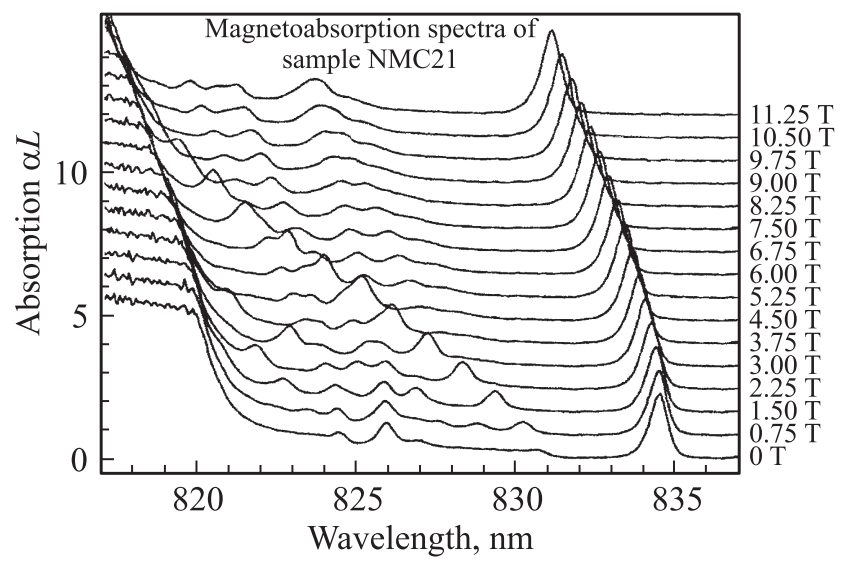

Рис. 5. Спектры поглощения одного из образцов (NMC21) в различных магнитных полях (перед удалением подложки).

Очень слабая сходимость веерной диаграммы легких дырок проявляется и в спектрах образцов с более высоким содержанием In (например, образец 4), но и здесь в экситонных спектрах легких дырок можно увидеть характерную дублетную структуру. Отметим также, что иногда слабая эквидистантная „тонкая структура“ обнаруживается в образцах высокого качества с малым содержанием In при энергиях выше дублета.

\section{5. Интерпретация „тонкой“ структуры экситонных переходов}

5.1. Перераспределение напряжений в с в ободных образцах. Здесь мы обсудим изменения в зонной структуре свободных образцов относительно образцов на подложке, возникающие из-за эффектов 

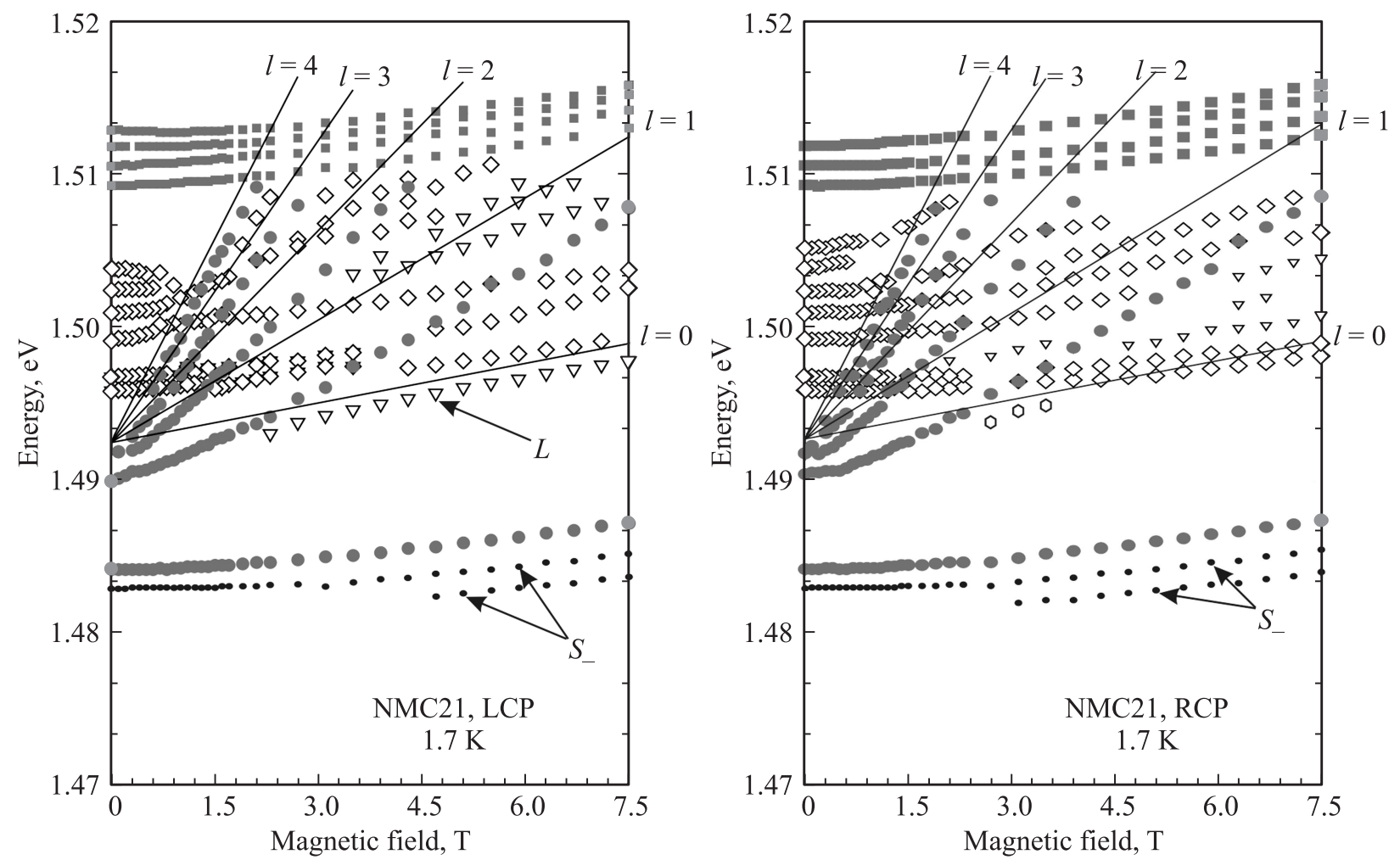

Рис. 6. Веерные диаграммы образца 3 в LCP- и RCP-поляризациях. Реконструированные положения переходов на уровни Ландау показаны линиями. $l$ обозначает квантовые числа Ландау. Прямоугольники для первых максимумов барьера GaAs, кружки для HH1E1, алмазы для LH1E1 и LH3E1 и треугольники для дополнительных переходов в веерных диаграммах - системы максимумов экситона LH-E1 в магнитном поле. S - дополнительные переходы, связываемые с монослойными отклонениями толщины слоя ямы.
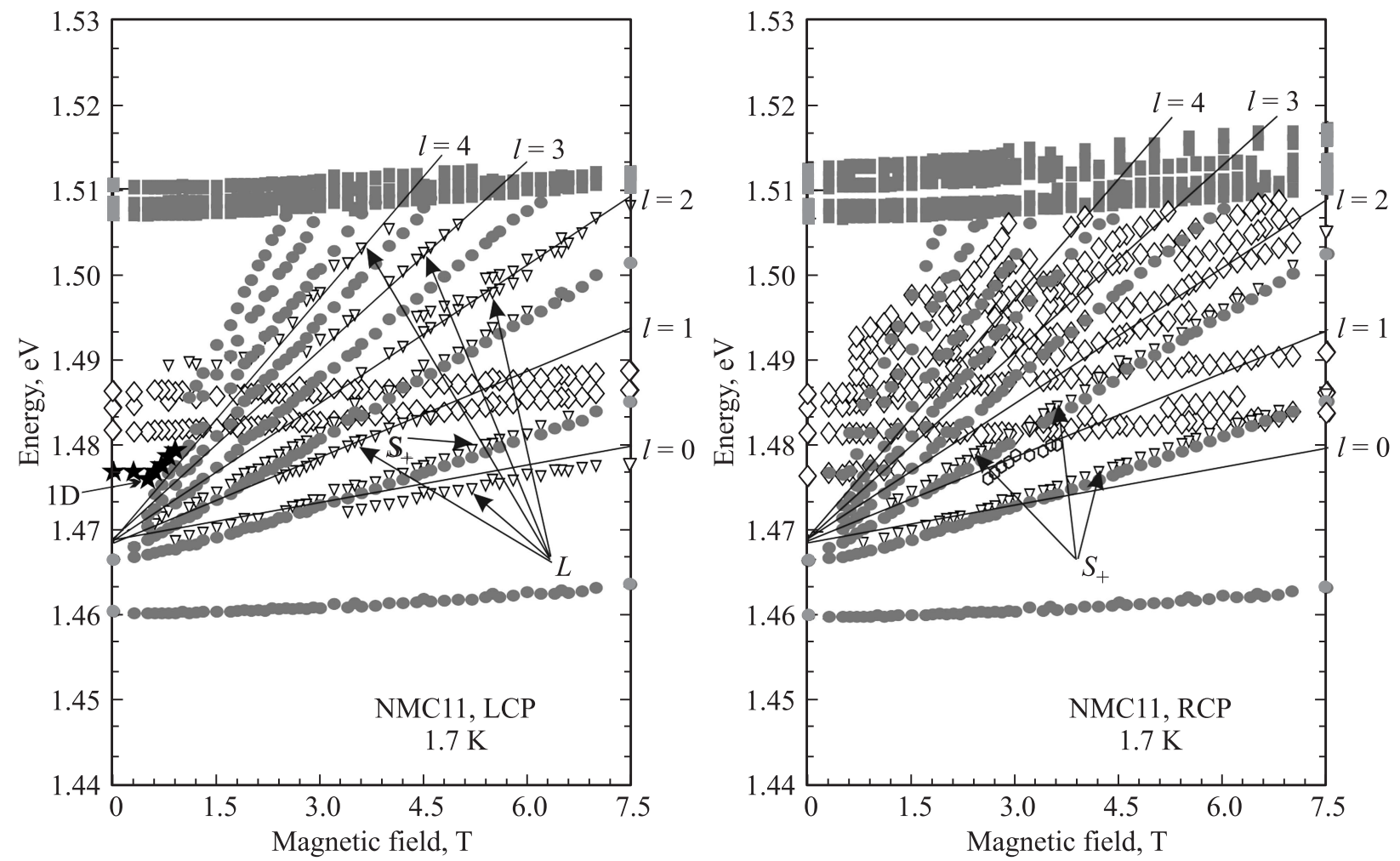

Рис. 7. Веерные диаграммы образца 4 в LCP- и RCP-поляризациях. L - дополнительные переходы, связываемые с возбужденными состояниями ДЭ, 1D - дополнительные переходы, предположительно - непрямые переходы на электронный уровень ямы. $\mathrm{S}_{+}$- дополнительные переходы, связываемые с монослойными отклонениями толщины слоя ямы. 
деформации. Экспериментально разница между спектрами поглощения свободных образцов и образцов на подложке огромна, как видно из рис. 3. В частности, наблюдаются существенные сдвиги линий, соответствующих тем же экситонным переходам. Для того, чтобы объяснить эти изменения, мы используем следующий формализм. Исходя из значений $\Delta e$ и $\delta E$, показанных в табл. 2, вычислим значение деформации $e$ в GaAs c использованием данных [27]

$$
\begin{aligned}
\Delta e & =2 b(1+\lambda) e=6.4 e, \\
\delta E & =a(2-\lambda) e=-9.7 e,
\end{aligned}
$$

что дает средние экспериментальные значения $e=4.3 \cdot 10^{-4}$ и $5.8 \cdot 10^{-4}$ для образцов 3 и 4 соответственно. Это означает, что параметр решетки $a$ образцов, выращенных на плоскости подложки GaAs, увеличивается: $a=a_{0}+\Delta a$, и это вызывает уменьшение величины деформации $e_{1}$ в слоях (In, Ga)As следующим образом:

$$
e_{1}=e_{0}+e\left(a_{0} / a_{1}\right) .
$$

Здесь $e_{0}$ является исходной деформацией в слоях $(\mathrm{In}, \mathrm{Ga}) \mathrm{As}, a_{0}$ и $a_{1}$ являются постоянными решетки в свободных слоях GaAs и (In, Ga)As при заданном значении $x$ соответственно. Различия между деформацией в образцах на подложке и для свободных образцов могут быть аппроксимированы как $\Delta e=e_{1}-e_{0}=e k$, где $k=5.6512 /(5.6512-0.4 x) \approx 1$. Это позволяет писать: $\Delta_{1}=-a_{x}\left(2-\lambda_{x}\right) e-\left|b_{x}\right|\left(1+\lambda_{x}\right)|e|$,

$$
\begin{aligned}
\Delta_{2}= & -a_{x}\left(2-\lambda_{x}\right) e-\left|b_{x}\right|\left(1+\lambda_{x}\right) e \\
& \times\left[1+9\left|b_{x}\right|\left(1+\lambda_{x}\right)\left|\left(e_{0}+e_{1}\right)\right| / 4 \Delta_{0 x}\right] .
\end{aligned}
$$

Рассчитывая деформационное расщепление экситонных линий, мы не приняли во внимание квантование экситонных поляритонов [25], которое различным образом изменяет положения экситонных пиков легких и тяжелых дырок. Во всяком случае, согласие между расчетными и экспериментальными энергиями экситонных переходов (табл. 2) является вполне удовлетворительным.

5.2. Общие особенности спектров магнитопогго щения.Спектры пропускания, измеренные после удаления подложки, без магнитного поля и при $B=1-3$ Т для одного из наших образцов приведены на рис. 4. Относительно слабые магнитные поля, не сильнее, чем $1 \mathrm{~T}$, индуцируют хорошо выраженные осцилляции в спектрах поглощения. Наблюдается значительное число максимумов поглощения, иногда мы видим более 20 пиков только в спектральной области, ограниченной положением поглощения барьера. Это также является доказательством высокого качества наших образцов. Это осциллирующее поведение магнитопоглощения характерно для межзонного магнетопоглощения квантовых ям и наблюдалось ранее в системе $\mathrm{AlGaAs} / \mathrm{GaAs}$ (см, например, [26]). Оно наблюдается в наших образцах, когда критерий сильного поля $\beta=\hbar \Omega / 2 R_{0} \gg 1$ (где

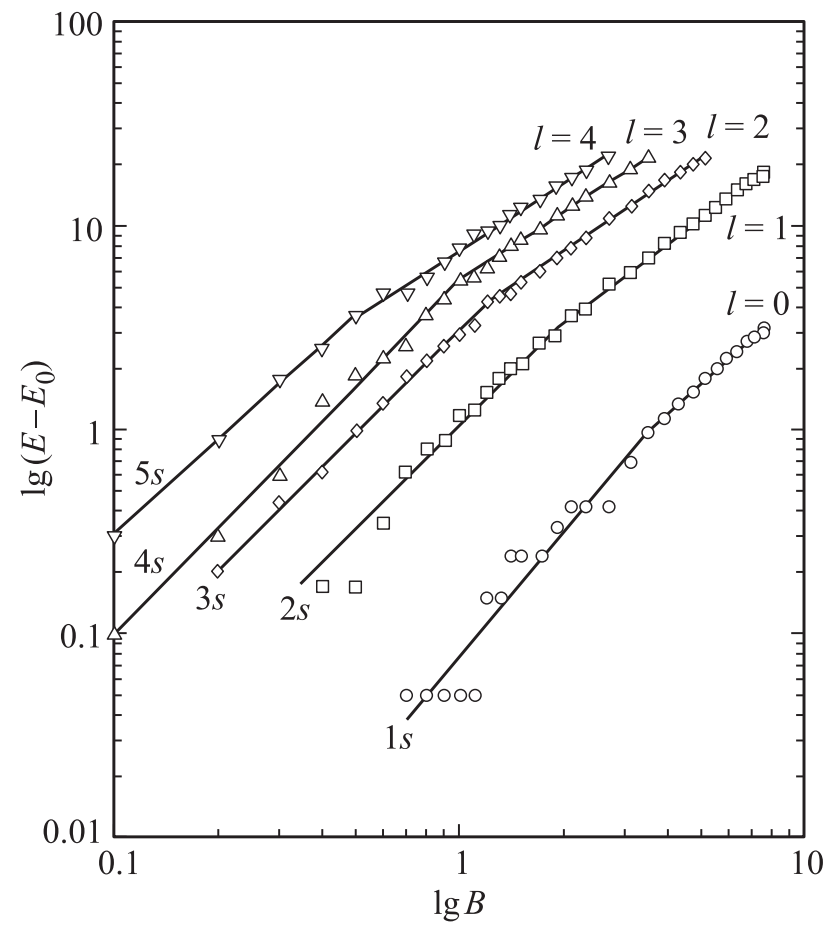

Рис. 8. Веерная диаграмма образца 3 в RCР-поляризации, построенная в логарифмическом масштабе.

$R_{0}$ - энергия связи экситона при $B=0$ и $\Omega$ представляет собой сумму циклотронных частот электронов и дырок) для основного состояния экситона еще не соблюден. Наблюдение осциллирующей структуры в этом случае является прямым следствием участия в поглощении возбужденных экситонных состояний. Однако следует отметить, что эти возбужденные состояния могут не быть четко различимыми при $B=0$, хотя время жизни экситона в этих состояниях должно быть достаточно большим, чтобы можно было обнаружить осцилляции поглощения в присутствии магнитного поля.

Веерные диаграммы (рис. 6) ясно показывают, что магнитоэкситоны с числами Ландау для электронов $l_{c}=1,2,3$ происходят из $2 s, 3 s, 4 s$ и $5 s$ возбужденных состояний экситона в квантовой яме соответственно (в этой связи см. рис. 8 для образца 3). Можно сделать вывод, что критерий сильного поля выполняется для этих возбужденных состояний при 1.8, 1.2, 0.9 и 0.5 T соответственно. Межзонный край поглощения в этом случае находится при $E_{N}=1.4925 \mathrm{eV}$. Резонно предположить, что все магнитоэкситоны с более высокими номерами Ландау соответствуют более высоким первоначальным возбужденным экситонным состояниям при $B=0$. Энергия связи основного состояния экситона HH1E1 оказывается $8.3 \mathrm{meV}$, а для $2 s$-состояния это $2.1 \mathrm{meV}$. Экстраполяция к нулевому полю дает энергии диссоциации $1.0,0.5$ и $0.3 \mathrm{meV}$ для $3 s, 4 s$ и $5 s$ возбужденных состояний соответственно. До некоторого критического поля $B \approx 6 \mathrm{~T}$ состояния магнитоэкситона испытывают параболический диамагнитный сдвиг (рис. 9). Только в 


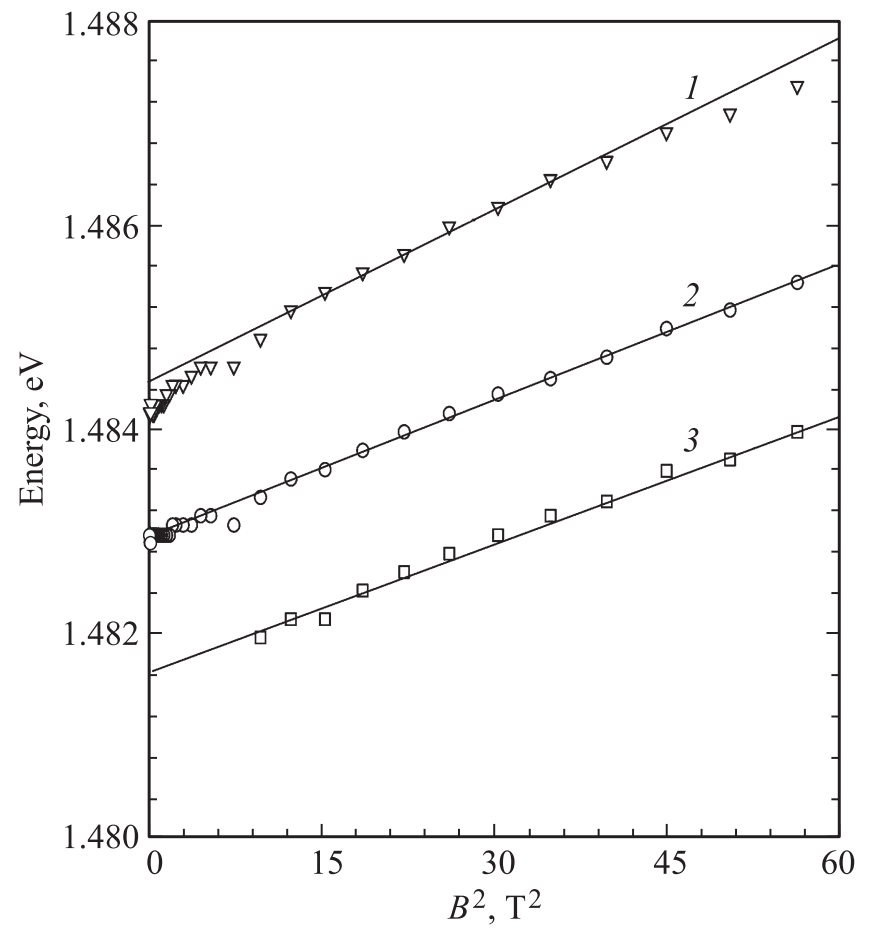

Рис. 9. Пики магнитопоглощения, зарегистрированные в окрестности перехода Е1НH1 в образце 3. Сплошные линии показывают рассчитанные энергии перехода Е1HН1 в QW толщиной $9 \mathrm{~nm} \mathrm{в} \operatorname{In}_{x} \mathrm{Ga}_{1-x} \mathrm{As} / \mathrm{GaAs}$, и в аналогичных QW, толще на один и два монослоя.

большем поле зависимость начинает приобретать квазилинейный наклон, характерный для уровней Ландау (предел „сильного поля““).

5.3. „Тонкая “ структура магнитопоглощения. Тонкие линии на рис. 6 показывают истинные положения для переходов между подзонами Ландау для переходов HН1E1 ( $l$ обозначает номер квантового числа Ландау). Наклон линий и точка их экстраполяции при $B=0$ характерны для состояний магнитоэкситона (DE), принадлежащих квантово-размерному состоянию HH1E1 нашей гетеросистемы [28,29]. Так что есть возможность связать $L$-линии с первым возбужденным состоянием серии DE, с квазиодномерным квантовым числом $v=1$. Интересно, что такие состояния, по всей видимости, проявляются в спектрах вблизи точки пересечения между основным состоянием DE и состоянием, принадлежащим следующему номеру Ландау, и краем диссоциации серии DE от предыдущего уровня Ландау. Эта особенность характерна для диамагнитного экситона в объемных кристаллах [30]. Существование $L$-линий только в LCP-спектрах можно понять, принимая во внимание правила отбора для возбужденных экситонных состояний [28,31]. Понятно, что появление таких состояний является свидетельством отклонения от двухмерности в нашей системе. Они должны отсутствовать в более тонких слоях QW.
Длинноволновые спутники к $1 s$-пикам магнитоэкситона, вероятно, связаны с энергетическим расщеплением экситонной линии за счет монослойных флуктуаций ширины QW. Такую тонкую структуру можно увидеть только тогда, когда характерный размер островков интерфейса с дополнительным монослоем превышает экситонный боровский радиус в плоскости ямы. Магнитное поле делает тонкую структуру, связанную с монослойными флуктуациями, более выраженной, что вполне естественно, так как оно эффективно уменьшает боровский радиус экситона в плоскости QW [32]. Именно поэтому второй сателлит появляется в более сильных полях (рис. 9). Расстояния между спутниками и основным состоянием экситона $\delta_{i}$ для образца 3 может быть оценено как: $\delta_{1}(0) \approx-0.8 \mathrm{meV}, \delta_{1}(7.5 \mathrm{~T}) \approx-1.6 \mathrm{meV}$ и $\delta_{2}(7.5 \mathrm{~T}) \approx-2 \mathrm{meV}$, при использовании приближенного соотношения

$$
\delta_{i}=\left(\Delta E+\alpha_{i} B^{2}\right) \delta L_{z} / L_{z}
$$

Здесь $\Delta E=\Delta E_{c}^{W}+\Delta E_{h h}^{W}-$ сумма энергий электронов и дырок, отложенных от дна зоны проводимости и от вершины валентной зоны соответственно, $\alpha_{i}$ представляет собой диамагнитный коэффициент сдвига, а $\delta L_{z}$ является изменением толщины слоя ямы из-за неоднородного заращивания поверхности. Расчетное значение $\delta_{1}(0)=0.7 \mathrm{meV}$ хорошо согласуется с экспериментальными расщеплениями между линиями при $B=0$. Отрицательный знак соответствует росту одиночных холмов на поверхности слоя. Несколько иначе ведет себя образец 4. Экситонные боровские радиусы, рассчитанные с использованием коэффициентов диамагнитного сдвига, полученных из рис. 9, составляют 9.7, 7.1 и $6.8 \mathrm{~nm}$ соответственно. Первый из них относится к основному экситонному состоянию ямы HH1E1 и имеет величину $\sim 10 \mathrm{~nm}$, полученную в результате вариационного расчета.

Радиус, соответствующий сателлитным пикам, оказывается заметно меньше, что указывает на то, что эти пики происходят из латерально ограниченных состояний ямы.

Дополнительные пики можно различить и на коротковолновой стороне основного экситонного пика. Их амплитуды возрастают с увеличением магнитного поля. Мы относим их к экситонным состояниям, связанным с монослойными сужениями QW. Было теоретически показано, что такие состояния, принадлежащие к двумерным континуумам экситонных состояний, имеют силу осциллятора того же порядка, что и локализованные экситоны [33]. Расщепление между основным пиком и его спутником, связанным с сужением QW на величину $\delta L_{c}$, можно оценить как

$$
\delta_{i}=\left[\Delta E+\hbar \omega_{0}(l+1 / 2)-R_{B, l}\right] \delta L_{z} / L_{z},
$$

где $\omega_{0}=e B / m c$ является циклотронной энергией свободного электрона, пропорциональной магнитному по- 
лю $B$, и $R_{B}$ есть соответствующая энергия связи. Значение $\delta L_{c}$, экстраполированное к нулевому полю, составляет около $0.85 \mathrm{meV}$. Относительные интегральные интенсивности основной линии и сателлитов дают возможность оценить концентрацию островков с расширением и сужением QW - с использованием формализма [33]. Но это тема отдельного исследования.

Последняя особенность, которую стоит обсудить, это спектральная линия, помеченная $1 D$ в веерной диаграмме образца 4 (рис. 7). Это слабая линия, которая наблюдается при энергиях выше, чем энергия экситонного перехода HН1E1 на $17 \mathrm{meV}$, что равно расстоянию между вершиной валентной зоны в барьере и квантовым уровнем тяжелых дырок НH1. Мы его относим к пространственно непрямым переходом барьерных дырок на электронный уровень в яме. Она исчезает, когда магнитное поле становится сильнее.

Следует отметить, что появление хорошо выраженных дискретных электронных и дырочных квантовых состояний в яме удивительно само по себе, если принять во внимание очень низкую концентрацию индия в наших образцах (около 3\% в QW тоньше, чем $10 \mathrm{~nm}$ ). В этом случае только один атом „примеси“ In находится в матрице GaAs на каждые две атомные цепочки, перпендикулярные плоскости ямы. Принимая во внимание случайное распределение атомов, можно ожидать существования областей с полностью GaAs атомными цепями, пересекающими весь слой QW. Тем не менее образцы характеризуются ультра-узкими спектральными линиями с практически ничтожным неоднородным уширением. Эта особенность наших образцов была рассмотрена отдельно в [29], и было показано, что (In, Ga)As/GaAs QW гетероструктуры с таким низким содержанием индия могут действительно рассматриваться как состоящие из бездефектного квазикристалла, обладающего к тому же достаточно совершенным интерфейсом. Наши исследования обнаруживают только (см. рис. 7,9) неоднородности в один-два монослоя.

В [20] была исследована температурная зависимость FWHM спектральных линий для HH1E1 состояния экситона, а также возможность образования экситонполяритонов при нормальном распространении световой волны через структуру с квантовыми ямами [20,34].

5.4. Опр еделение циклотронных масс НН1Е 1 экситона. Для того чтобы получить циклотронную массу экситона (приведенную массу относительного движения электрона и дырки), приходится реконструировать экспериментальную веерную диаграмму в диаграмму для переходов между уровнями Ландау, добавляя энергии связи экситона к энергиям пиков магнитопоглощения [28,29]. Для этого мы выполнили расчет энергий связи различных экситонных состояний в зависимости от магнитного поля, как показано на рис. 10. Для расчета состояний экситона на тяжелых дырках мы использовали вариационный подход, разработанный в [20]. Для расчета состояний экситона на тяжелых дырках с более высокими числами Ландау

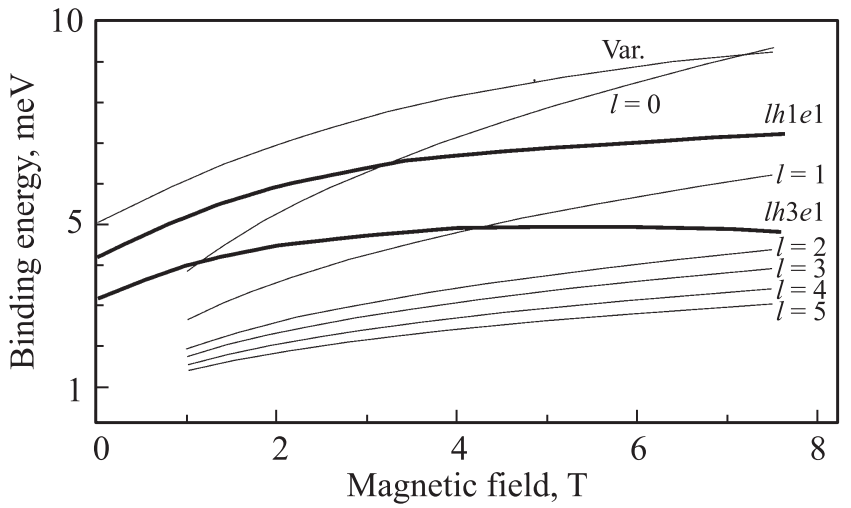

Рис. 10. Энергии связи для экситонов на тяжелых дырках (тонкие линии) и легких дырках (жирные линии). „Var“ обозначает результат вариационного расчета для основного состояния тяжелых дырок. Все другие тонкие кривые были построены при помощи расчета по теории возмущений [20], в то время как полужирные кривые были рассчитаны при помощи самосогласованной вариационной техники с учетом эффекта CW (как описано в тексте).

мы применили метод теории возмущений [20], в то время как экситонные состояния на легких дырках были вычислены с помощью самосогласованного вариационного метода с учетом эффекта $\mathrm{CW}$, как описано в разд. 2 .

Все кривые для переходов между подзонами Ландау, в том числе и для первой подзоны Ландау с $l=0$, сходятся к одной энергии при $B=0$ в пределах небольшой ошибки. Это подтверждает хорошую точность расчета энергий связи магнитоэкситона (DE). На рис. 11 показана реконструкция веерных диаграмм Ландау с использованием вычисленных энергий связи экситона в магнитном поле. Реконструированные веерные диаграммы Ландау в единицах $E_{l l}=0.116 B(l+1 / 2)$ позволяют собрать все экспериментальные точки, принадлежащие к различным состояниям по числу Ландау в одну кривую, как это можно увидеть на рис. 11. Такое построение позволяет оценить приведенные эффективные массы $\mu$ электрона и дырки в плоскости квантовой ямы из наклона реконструированной прямой для переходов между подзонами Ландау $\Delta E / \Delta B$. При этом $0.116=\hbar \omega_{e}^{c} / B-$ это циклотронная энергия свободного электрона на единицу напряженности магнитного поля $B$.

К сожалению, здесь у нас нет возможности экспериментально разделить вклады электрона и тяжелой дырки в полученных приведенных массах. Более того, данные, которые мы обсуждаем, получены для образцов с различной шириной квантовой ямы $L_{z}$.

Не параболичность дисперсионных кривых для электронов в нашей модели немного отличается для разных величин $L_{z}$. Массы тяжелых дырок соответствуют известным зависимостям от концентрации индия для твердых растворов (например, $m_{c}^{*} m_{0}^{-1}=0.0660-0.0537 x$ $+0.0116 x^{2}$ из [31]). Мы получили зависимость эффек- 


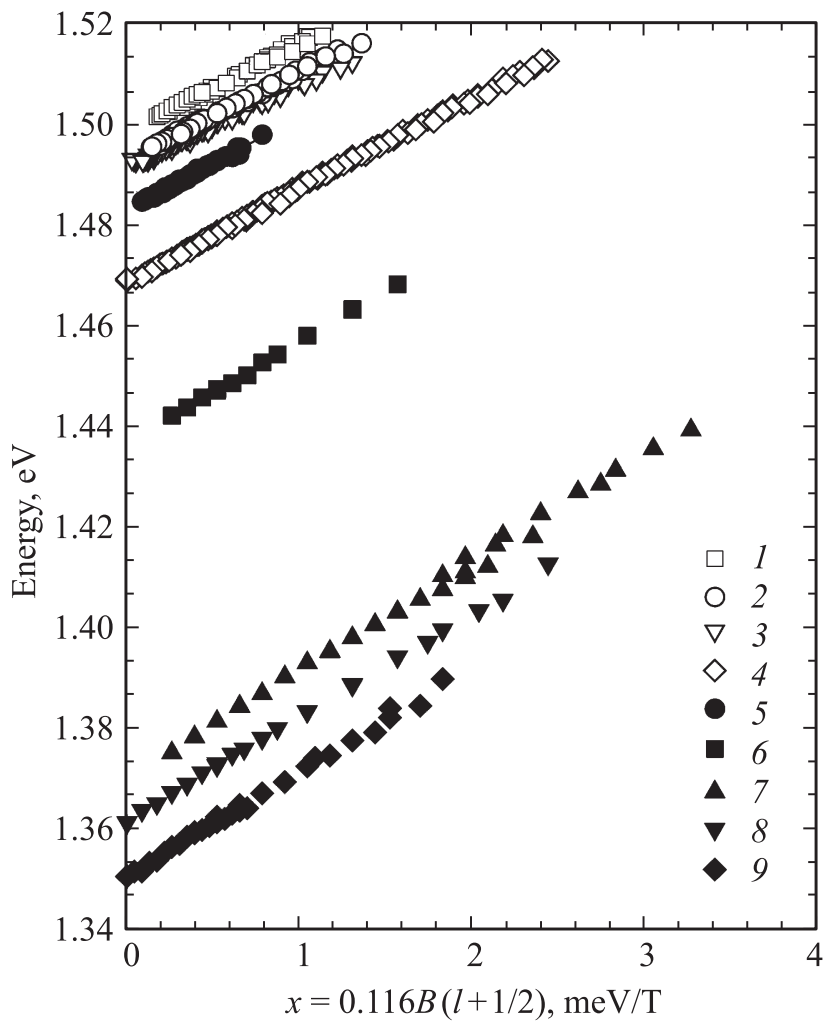

Рис. 11. Положения уровней энергии для всех наших образцов из табл. 1 , скорректированные с учетом энергии связи $R_{B}$ и построенные как функция $x=\hbar \omega_{0}(l+1 / 2)$, где $l-$ номер квантового уровня Ландау.

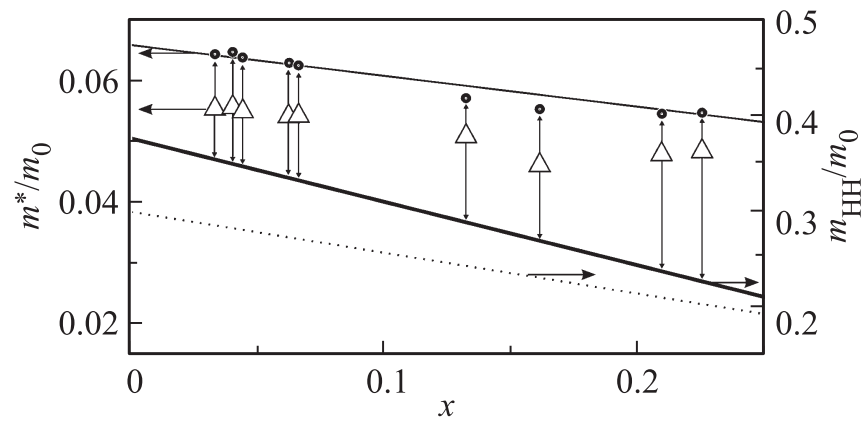

Рис. 12. Оценочные эффективные массы электронов (левая шкала) и тяжелых дырок (правая шкала) в гетероструктурах (In, Ga)As/GaAs c QW в зависимости от концентрации In в ямах.

тивной массы тяжелых дырок от содержания индия

$$
m_{0}^{-1} m_{h h}^{*}(x)=0.385-0.88 x+0.86 x^{2}
$$

(см. рис. 12). Можно видеть, что масса тяжелой дырки для первого уровня квантования (НH1) в (In, Ga)As/GaAs намного тяжелее, чем в ненапряженных QW. Сравнивая это выражение с зависимостью тяжелых дырок от $x$ в объемном материале (например, с $\left.M_{h h}(x)=0.445-0.035 x[18]\right)$ и для массы дырок в $\mathrm{QW}$, рассчитанной по формулам $(13,14)$, мы приходим к следующим выводам: (a) не существует никакого существенного смешивания легких и тяжелых дырок в QW наших структур, (b) экспериментальные данные находятся в хорошем согласии с теорией [24]. В частности, теоретическое предсказание, что тяжелые дырка сохранят свою тяжелую эффективную массу в плоскости QW вследствие проникновения легких дырок в барьерные слои, подтверждается. Аналогичное наблюдение было сделано в [35] для QW в $\operatorname{In}_{x} \mathrm{Ga}_{1-x} / \mathrm{As} / \mathrm{GaAs}$ с $x=0.1$ и $L_{Z}=2.5-20 \mathrm{~nm}$, где масса тяжелых дырок оказалась в диапазоне $0.19-0.25 m_{0}$.

5.5. Экситонные переходы легких дырок. Как уже отмечалось ранее, экситонные переходы легких дырок ведут себя в магнитном поле очень специфически по сравнению с типичным поведением магнитоэкситона в QW типа I, или с поведением экситонов на тяжелых дырках в наших гетероструктурах. Такое поведение может быть понято в рамках модели $\mathrm{CW}$, сформулированной выше. На рис. 13 приводится профиль CW, рассчитанный вариационно по формуле (8). Там же показаны энергетические положения так называемых осцилляторных уровней. Только уровни, имеющие четные индексы, являются оптически активными, если рассматривать переход в низшее состояние электронов в QW. Глубина CW для наших образцов, по-видимому, находится в пределах $13-16 \mathrm{meV}$. Энергетический зазор, разделяющий два уровня легких дырок с $n=0$ и $n=2$, составляет $5.8 \mathrm{meV}$ для образца 4 и $5.2 \mathrm{meV}$ для образца 3. Для получения положений осцилляторных уровней приходится учитывать энергии связи экситона, рассчитанные в соответствии с уравнением (7) (см. рис. 10). Это дает нам возможность восстановить истинные положения осцилляторных уровней и их зависимости от магнитного поля (см. ниже линии $C_{1}$ и $C_{2}$ на рис. 17). При $B=0$ они отделены друг от друга $3.8 \mathrm{meV}$ и $3.1 \mathrm{meV}$

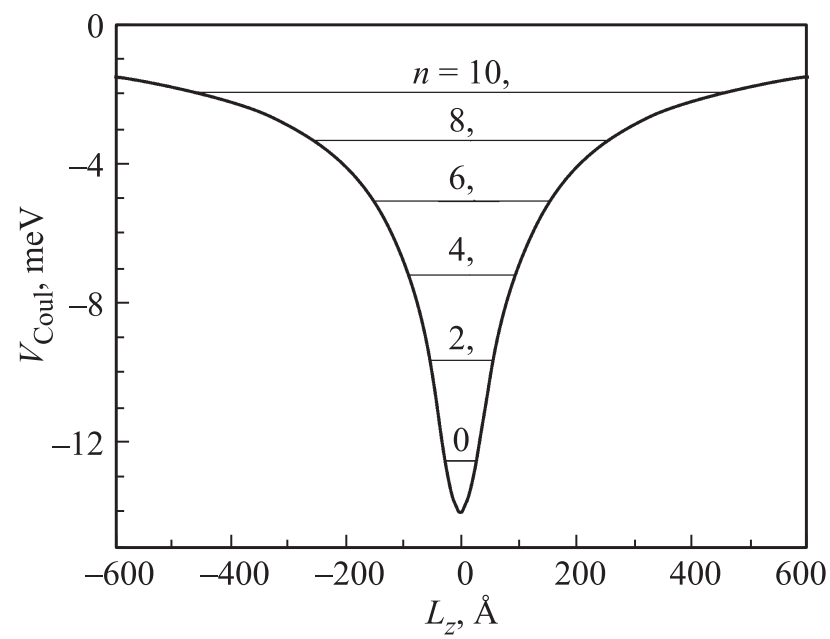

Рис. 13. Рассчитанный $\mathrm{CW}$-профиль для легкой дырки в гетеросистеме $\mathrm{In}_{0.05} \mathrm{Ga}_{0.95} \mathrm{As} / \mathrm{GaAs} \mathrm{c} \mathrm{QW}$-толщиной $8 \mathrm{~nm}$. Показаны энергии четных „осцилляторных“ состояний легких дырок в $\mathrm{CW}$-потенциале. 
для образцов 4 и 3 соответственно. Эти значения вполне соответствуют экспериментальным данным (см. рис. 13). Небольшие отклонения можно связывать с неопределенностью в эффективных массах легких дырок, которые различны для разных квантовых состояний.

В целом поведение пиков, связанных с экситонами на легких дырках, в магнитном поле сильно отличается от поведения пиков экситоннов на тяжелых дырках.

\section{6. Обсуждение}

6.1. „Радиальные“ и „„СW“ части энергии связи экситона. В принятом здесь адиабатическом приближении, можно разделить вклады в энергию связи экситона (7), происходящие от движения электрона и дырки в плоскости ямы (далее упоминается как радиальная часть энергии связи) и от локализация дырок в $\mathrm{CW}$ (далее упоминается как CW-часть энергии связи). Радиальная часть $E_{R}$ определяется уравнением

$$
\begin{aligned}
\left\{-\frac{\hbar^{2}}{2 \mu} \frac{1}{\rho}\left(\rho \frac{\partial}{\partial \rho}\right)\right. & \left.-\frac{e^{2}}{\varepsilon} \iint d z_{e} d_{h} \frac{U_{e}^{2}\left(z_{e}\right) U_{h}^{2}\left(z_{h}\right)}{\sqrt{\rho^{2}+\left(z_{e}-z_{h}\right)^{2}}}\right\} \\
& \times f(\rho)=E_{R} f(\rho),
\end{aligned}
$$

в то время как уравнение для $\mathrm{CW}$-части выглядит следующим образом:

$$
E_{\mathrm{CW}}=E_{\text {net }}-E_{\mathrm{tot}},
$$

где $E_{\text {net }}$ и $E_{\text {tot }}$ даются уравнениями

$$
\begin{gathered}
\left\{-\frac{\hbar^{2}}{2 m_{h}} \frac{\partial}{\partial z_{h}}+V_{h}\left(z_{h}\right)\right\} U_{h}\left(z_{h}\right)=E_{\mathrm{net}} U_{h}\left(z_{h}\right) \\
\left\{-\frac{\hbar^{2}}{2 m_{h}} \frac{\partial}{\partial z_{h}}+V_{h}\left(z_{h}\right)+V_{\mathrm{CW}}\left(z_{h}\right)\right\} U_{h}\left(z_{h}\right)=E_{\mathrm{tot}} U_{h}\left(z_{h}\right) .
\end{gathered}
$$

В обычном первом типе QW радиальная часть доминирует над CW-частью в отличие от QW-типа II. Радиальная часть является гладкой и хорошо известной функцией ширины и высоты барьера QW. Наоборот, вклад CW, насколько нам известно, специально не анализировался. Представляется полезным выполнение такого рода анализа для нашей системы. Кроме того, мы получим энергии $\mathrm{CW}$ в зависимости от содержания индия в наших образцах, что позволят также экспериментально определить глубину кулоновской ямы $V_{0}$.

6.2. Глубин а кулоновской ямы. Мы можем оценить глубину CW на наших образцах в зависимости от содержания индия $x$, из данных магнитопоглощения и данных рентгеновской дифракции. Сравним зависимости положения энергии экситонного пика тяжелых и легких дырок от содержания индия. Если мы будем игнорировать кулоновскую яму, соотношения этих энергий могут быть записаны следующим образом:

$$
E_{H H 1 E 1}=E_{g}(x)+E_{g}^{H}(x)-\Delta_{e}(x) / 2+\Delta E_{E 1}^{W}+\Delta E_{H H 1}^{W}-R_{h}
$$

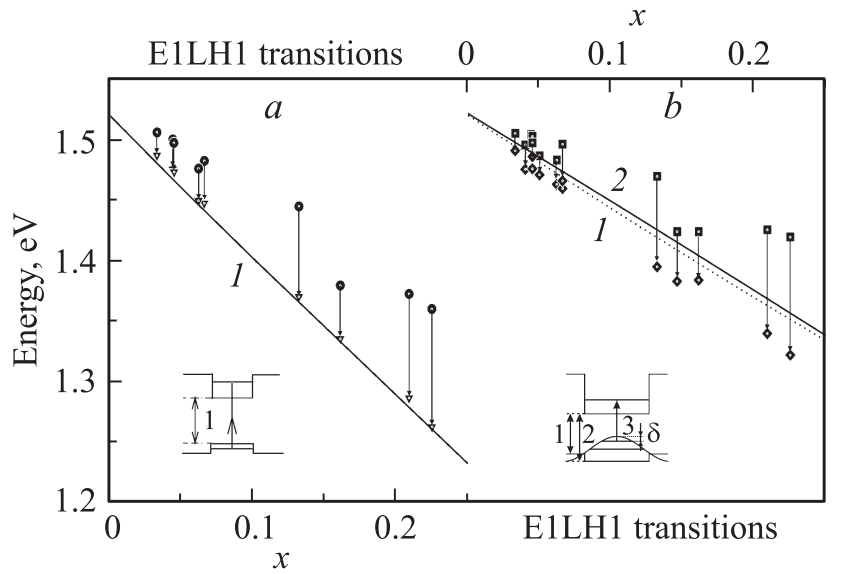

Рис. 14. Оценка глубины $\mathrm{CW}$ из экспериментальных энергий экситонного перехода тяжелых $(a)$ и легких дырок $(b)$. Энергии переходов построены в зависимости от содержания индия $x$ и сравниваются с расчетными энергиями прямых и непрямых экситонных переходов (как показано на вставках). Стрелки показывают перенормировки экспериментальных энергий переходов путем вычитания энергий квантования электронов и дырок.

для перехода НH1E1 и

$$
E_{L H 1 E 1}^{U B}=E_{g}(0)+\Delta E_{c}(x)-\Delta E_{c}^{H}(x)+E_{l h}+\Delta E_{E 1}^{W}-R_{l}^{U B}
$$

для перехода LH1E1 с легкой дыркой на краю запрещенной зоны GaAs (фактически это надбарьерное состояние легкой дырки, т.к. наша структура принадлежит к типу II). В случае пространственно-непрямых переходов LH1E1 (с легкой дыркой на краю зоны GaAs) получим

$$
E_{L H 1 E 1}^{(S I)}=E_{g}(0)+\Delta E_{c}(x)-\Delta E_{c}^{H}(x)+\Delta E_{E 1}^{W}-R_{l}^{S I} .
$$

Здесь $\Delta E_{E 1}^{W}, \Delta E_{H H 1}^{W}$ и $\Delta E_{l h}$ и $\Delta E_{c}^{H}(x), \Delta E_{c}^{H}(x)$ представляют энергии квантовых уровней электронов и тяжелых дырок, измеренные от дна электронной зоны и вершины подзоны тяжелых дырок соответственно, $\Delta E_{c}(x)$ является разрывом зоны проводимости и $R_{h}, R_{l}^{U B}$ и $R_{l}^{S I}$ являются энергиями связи HH1E1, LH1E1 надбарьерных и пространственно-непрямых экситонов соответственно.

Так как в наших образцах содержание индия и толщины QW различны, энергии $\Delta E_{E 1}^{W}$ и $\Delta E_{H H 1}^{W}$ изменяются от образца к образцу. Мы вычли эти две энергии из экспериментально измеренных резонансных уровней HH1E1, как показано на рис. 14, $a$. Можно видеть, что энергии переходов, скорректированные таким образом, могут быть соединены прямой линией, которая соответствует зависимости ширины запрещенной зоны от содержания индия в деформированном (In, Ga)As. Данные взяты из рис. 1 и скорректированная с учетом радиальной части энергии связи экситона (20). Отклонения между точками и линией не превышают $1-2 \mathrm{meV}$. Это означает, что для экситона на тяжелых дырках $\mathrm{CW}$ часть энергии связи ничтожно мала. 


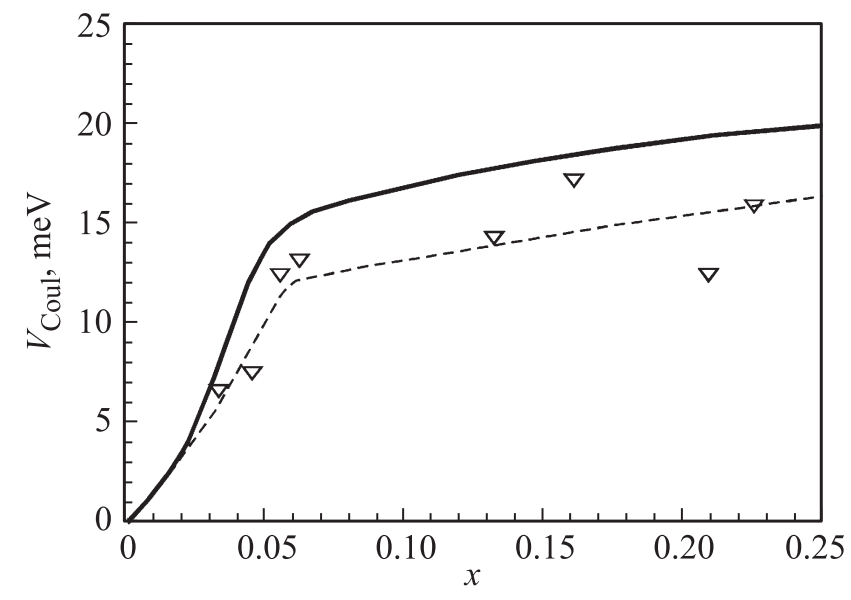

Рис. 15. Зависимость CW-части энергии связи экситона на легких дырках от концентрации индия $x$, полученная из экспериментальных положений пиков (треугольники), так же, как на рис. 14, и рассчитанная глубина CW (пунктирная линия). Сплошная линия показывает расчетную глубину CW.

Та же процедура перенормировки экспериментальных энергий экситона была применена в случае экситона на легких дырках (рис. 14, b). Кривые (1) и (2) показывают ширину запрещенной зоны в (In, Ga)As, энергию перехода между дном зоны проводимости в (In, Ga)As и верхней частью валентной зоны в GaAs как функцию содержания индия соответственно. Обе энергии скорректированы на радиальную часть энергии связи экситона. Можно видеть, что перенормированные экспериментальные энергии экситонных переходов лежат значительно ниже, чем обе эти кривые. Мы связываем эту разницу с энергией локализации первого осцилляторного состояния $n=0$ в $\mathrm{CW}$

$$
E_{l}^{U B}-E_{\exp } \approx E_{C W}=E_{n}(n=0)-V_{0},
$$

где $E_{n}(n=0)$ - глубина первого осцилляторного состояния, измеренная от нижней части CW. На рис. 15 показаны экспериментальные значения $\Delta$ как функция $x$. На этом же рисунке глубина $V_{0} \mathrm{CW}(x)$, вычисленная для 8-nm QW, показана сплошной линией. Величина $V_{0}$, по-видимому, сильно зависит от $x$ при малых значениях концентрации индия. Это происходит потому, что глубина $\mathrm{CW}$ чувствительна к локализации функции электронной огибающей в направлении оси роста, которая сильно зависит от высоты барьера при малых $x$. Дальнейшее увеличение $x$ не вызывает сколько-нибудь значительного сдавливания электронной огибающей, так что $V_{0}(x)$ насыщается. В этом пределе из экспериментальных данных мы получаем

$$
E_{C W} \approx 12-16 \mathrm{meV}
$$

Используя расчетное значение $E_{n}(n=0) \approx 2-3 \mathrm{meV}$, можно оценить глубину $\mathrm{CW}$ при $x>0.05$ как 14-19 meV. Это, как представляется, в хорошем согла- сии с расчетным значением (сплошная линия в рис. 15). Обратим внимание, что $\mathrm{CW}$-вклад в энергию связи экситона на легкой дырке оказывается очень важным в большей части наших образцов. $\mathrm{CW}$-эффект позволяет экситонам в квантовых ямах типа II иметь энергию связи, приближающуюся и даже превышающую 2D-предел. Превышение двумерного предела на является парадоксом, если принять во внимание определение энергии связи как разницы между энергией невзаимодействующей электрон-дырочной пары в квантовой яме и энергией экситона. В квантовой яме второго типа энергия дырки в материале барьера ниже, чем в материале ямы. За счет этой разницы экситон может иметь энергию, отстоящую от энергии электрон-дырочной пары на величину, превосходящую двумерный предел.

6.3. Осцилляторные уровни кулоновской ямы. Спектры магнито-поглощения образцов с малыми $x$ показывают богатую мультипиковую структуру экситонных переходов на легких дырках (рис. 16). А именно, кроме первых двух интенсивных максимумов, связанных с E1LH1 и E1LH3 экситонными состояниями, можно наблюдать ряд эквидистантных пиков (см. также рис. 17, где показаны только переходы, связанные с экситонами на легких дырках). Аналитическое выражение для энергий осцилляторных уровней экситона в кулоновской яме можно найти в [8]

$$
E_{n}=-\frac{\hbar^{2} \alpha^{2}}{8 m_{h}}\left[-(1-2 n)+\sqrt{1+\frac{8 m_{h} V_{0}}{\hbar^{2} \alpha^{2}}}\right]
$$

Параметры $V_{0}$ и $\alpha$ найдены в результате численной аппроксимации точного CW-потенциала (8) с помощью

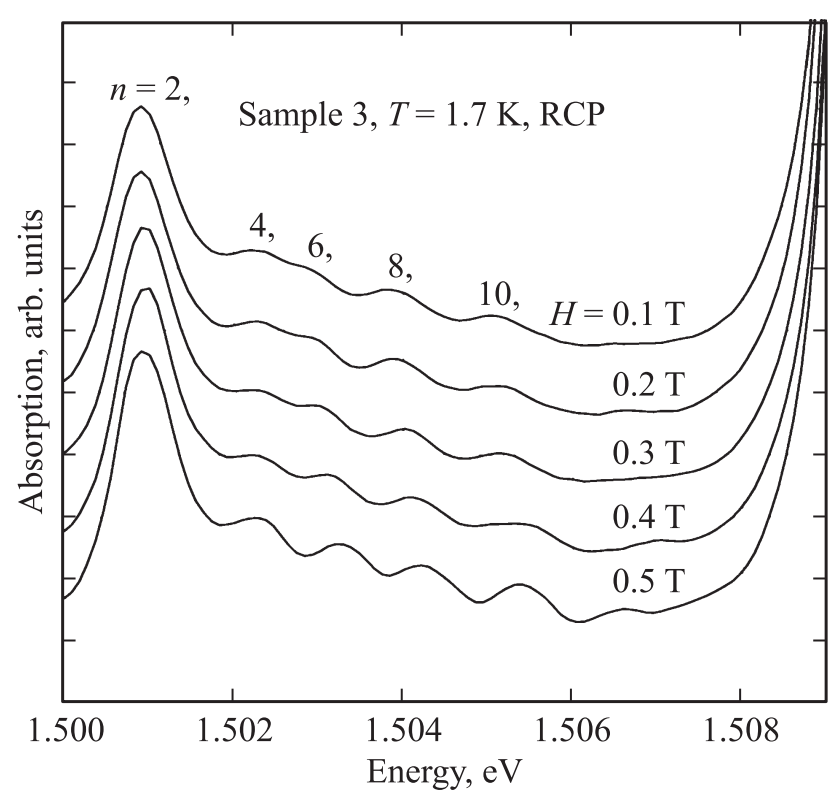

Рис. 16. Надбарьерные осцилляторные уровни CW, зарегистрированные в спектрах поглощения в структурах (In, Ga)As/GaAs c QW (образец 3) при различных значениях магнитного поля. 

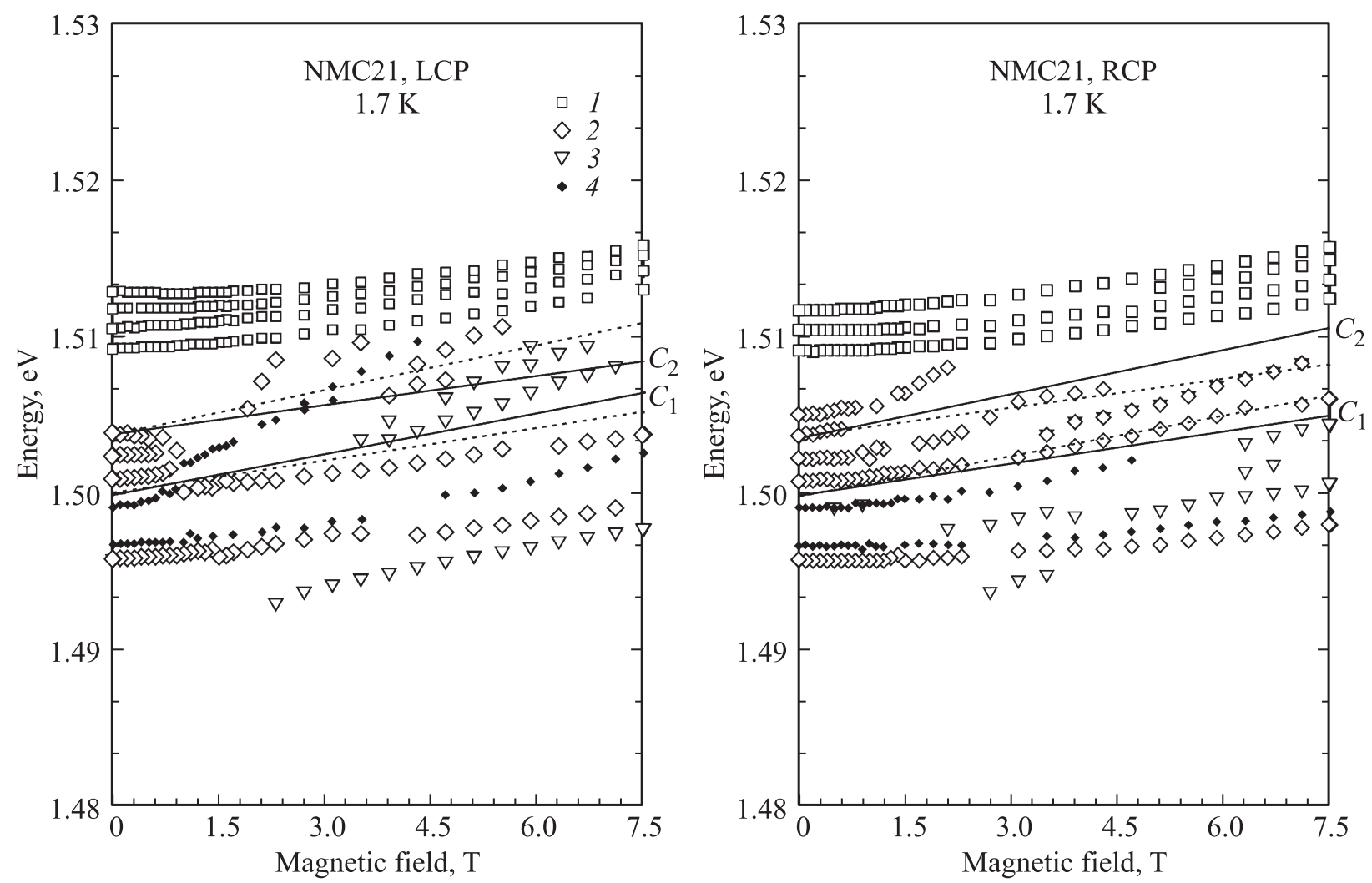

Рис. 17. Веерные диаграммы для экситонных переходов легких дырок в образце 3 , LCP- и RCP-спектры. Переходы экситона с тяжелой дыркой исключены. 1 - экситонные состояния в барьерах GaAs, 2 - переходы экситонов с легкой дыркой, 3 - переходы возбужденных состояний с меньшим числом Ландау $l, 4$ - дополнительная структура, связанная с квантованием состояний легкой дырки, $\mathrm{C}_{1,2}$ и сплошные линии - положения осцилляторных уровней $n=0,2$.

функции (9). Величина $\alpha$ имеет значение порядка обратного боровского радиуса экситона: $\alpha=8.5 \cdot 10^{-3} \AA^{-1}$ для $L_{Z}=80 \AA$ и $x=0.1[9]$. Зная $\alpha$ и $V_{0}$, можно найти эффективную массу легких дырок из экспериментальной разницы между ближайшими уровнями. При $n=2$ мы получим $m_{l h}=0.0826 m_{0}$, что практически идентично массе легких дырок в объемном GaAs. Это естественно, если иметь в виду весьма существенное проникновение волновой функции легких дырок в барьеры. Следует отметить, что для образца 3 глубина $\mathrm{CW}$ не превышает $5 \mathrm{meV}$ (см. 15). Именно поэтому экситонные состояния с $n>2$ делокализованы и представляют собой своего рода надбарьерные экситонные резонансы. Эти состояния могут иметь существенную силу осциллятора, как было показано теоретически в работе [28].

Множество пиков в измеренных спектрах магнитопоглощения наших образцов не могут быть однозначно связаны с одним из экситонных переходов, описываемых нашей моделью. Тонкая структура экситонов в квантовых ямах второго типа в магнитном поле чрезвычайно богата и для ее полной интерпретации необходимы новые эксперименты и более детальный теоретический анализ.

Кроме того, заметим, что за последние годы к гетеросистеме $(\mathrm{In}, \mathrm{Ga}) \mathrm{As} / \mathrm{GaAs}$ с квантовыми ямами неод- нократно с успехом возвращались как для решения прикладных задач, так и с рассмотрением фундаментальных аспектов физики конденсированного состояния. Так, в [36] были получены, повидимому, наиболее совершенные слои $\operatorname{In}_{x} \mathrm{Ga}_{1-x} \mathrm{As}$, обладающие минимальной шириной линии основного экситонного состояния, и при изучении динамики релаксации экситонного состояния - обнаружено необычное поведение экситонного поляритона, а именно очень узкий по величине волнового вектора диапазон его взаимодействия с фононами и другими рассеивателями. В [37] на базе квантовых ям в $\operatorname{In}_{x} \mathrm{Ga}_{1-x}$ As был создан мощный инжекционный лазер с уширенным спектром стимулированного излучения, а в [38] сообщается о создании высокоэффективных гетероструктур с квантовыми ямами II-го типа на базе $(\mathrm{GaIn}) \mathrm{As} / \mathrm{Ga}(\mathrm{AsSb})$. Эффект кулоновской ямы обсуждался в $[39,40]$. Эти работы подтверждают актуальность данного исследования, проливающего свет на физику экситонных состояний в этой гетеросистеме.

\section{7. Заключение}

1. Мы привели основные результаты магнитооптического исследования высококачественных гетероструктур 
(In, Ga)As/GaAs с квантовыми ямами. После удаления подложки методом химического травления нам удалось пронаблюдать богатую тонкую структуру экситонных переходов с тяжелыми и легкими дырками. Прикладывая магнитные поля до 7 и $12 \mathrm{~T}$, мы наблюдали хорошо выраженные веерные диаграммы для экситонов на тяжелых дырках и обнаружили их отсутствие для экситонов на легких дырках. Нам удалось обнаружить богатую тонкую структуру возбужденных экситонных состояний, особенно в случае экситонов на легких дырках.

Магнитооптические данные были проанализированы с использованием нескольких методов расчета, а именно, теории Кона-Латтинжера, обобщенной для расчетов валентных подзон в напряженных квантовых ямах смешанного типа: типа I для тяжелых дырок и типа II для легких дырок, самосогласованного вариационного расчета экситонных состояний, учитывающего эффект кулоновской ямы, теории возмущений применительно к диамагнитным экситонам в сильных магнитных полях. Нам удалось получить очень хорошее согласие между теорией и экспериментом и интерпретировать большую часть пиков в спектрах поглощения.

2. Наши основные общие выводы по обсуждаемой работе заключаются в следующем.

2.1. (In, Ga)As QW с очень низким содержанием индия $(<3 \%)$ представляют собой бездефектной квазикристалл, состоящий из однородных $2 D$-слоев. Высокое качество структур видно по очень малому уширению спектральных линий экситонных состояний, что позволяет наблюдать впечатляющее число возбужденных состояний экситона.

2.2. В отличие от предсказаний традиционной теории Кона-Латтинжера для QW с бесконечными барьерами, в структурах смешанного I-II-типов дырочные подзоны характеризуются объемными значениями эффективных масс. Это является следствим значителоьного проникновения волновой функции легкой дырки в барьеры.

2.3. CW-эффект определяет тонкую структуру спектров экситонов на легких дырках. В частности, это приводит к эффективному увеличению энергии связи экситонов на легких дырках. Энергия связи даже превышает двумерный предел в QW с $x>10 \%$. Кроме того, в спектрах поглощения из-за квантования легких дырок в потенциале $\mathrm{CW}$ появляется характерная осцилляторная структура.

2.4. $g$-фактор состояния LH3 имеет противоположный знак по отношению к $g$-фактору основного состояния легкой дырки. Этот эффект, вероятно, вызван более сильным проникновением волновой функции LH3 в барьеры по сравнению с волновой функцией LH1, однако он все еще нуждается в детальной теоретической интерпретации.

Авторы считают своим долгом высказать благодарность Х.М. Гибсу и Г. Хитровой за изготовление и предоставление исходных образцов и проведение измерений оптического пропускания в поле $11 \mathrm{~T}$, а также хотели бы подчеркнуть, что именно их высокое качество предопределило успех работы. Авторы благодарны С.И. Кохановскому, выполнившему подготовку образцов и значительную часть спектроскопических измерений, а также Н.Д. Ильинской, осуществившей удаление подложек.

\section{Список литературы}

[1] J.P. Reithmaier, R. Hoger, H. Riechert, A. Heberle, G. Abstreiter, G. Weimann. Appl. Phys. Lett. 56, 536 (1990).

[2] X.M.Fang, X.C.Shen, H.Q.Hou, W.Feng, J.M.Zhou, F.Koch. Surf. Sci. 228, 351 (1990).

[3] Y.S. Huang, H. Qiang, F.H. Pollak, G.D. Pettit, P.D. Kirchener, J.M. Woodall, H. Stiager, L.B. Sorensen. J. Appl. Phys. 70, 7537 (1991).

[4] F.S. Zhang, H. Luo, N. Dai, N. Samarth, M. Dobrowolska, J.K. Furdyna. Phys. Rev. B 47, 3806 (1993).

[5] J. Leymarie, C. Monier, A. Vasson, A.-M. Vasson, M. Leroux, B. Courboulès, N. Grandjean, C. Deparis, J. Massies. Phys. Rev. B 51, 13274 (1995).

[6] P. Disseix, J. Leymarie, A. Vasson, A.-M. Vasson, C. Monier, N. Grandjean, M. Leroux, J. Mas. Phys. Rev. B 55, 2406 (1997).

[7] J. Leymarie, P. Disseix, M. Rezki, C. Monier, A. Vasson, A.M. Vasson. Mater. Sci. Eng. B 44, 147 (1997).

[8] R.P. Seisyan, A.V. Kavokin, S.I. Kokhanovskii, A.I. Nesvizhskii, M.E. Sasin, M.A. Sinitsin, B.S. Yavich. Semicond. Sci. Technol. 10, 611 (1995).

[9] А.В. Кавокин, С.И. Кохановский, А.И. Несвижский, М.Э. Сасин, Р.П. Сейсян, В.М. Устинов, А.Ю. Егоров, А.Е. Жуков, С.В. Гупалов. ФТП 31, 1109 (1997).

[10] Ал.А. Эфрос. ФТП 20, 128 (1986).

[11] G. Peter, E. Deleporte, G. Bastard, J.M. Berroir, C. Delalande, B. Gil, J.M. Hong, L.L. Chang, J. Lumin. 52, 147 (1992).

[12] A.V. Kavokin, V.P. Kochereshko, G.R. Pozina, I.N. Uraltsev, D.R. Yakovlev, G. Landwehr, R.N. Bicknell-Tassius, A. Waag. Phys. Rev. B 46, 9788 (1992).

[13] A.V. Kavokin, M.A. Kaliteevski, S.V. Goupalov, J.D. Berger, O. Lyngnes, H.M. Gibbs, G. Khitrova, A. Ribayrol, A. Bellabchara, P. Lefebvre, D. Coquillat, J.P. Lascaray. Phys. Rev. B 54, R11078 (1996).

[14] А.И. Екимов, А.А. Онущенко, Ал.Л. Эфрос. Письма в ЖЭТФ 43, 292 (1986).

[15] A.I. Ekimov, Al.L. Efros, M.G. Ivanov, A.A. Onushchenko, S.K. Shumilov. Solid State Commun. 69, 565 (1989).

[16] D.J Arent, K. Deneffe, C. Van Hoof, J. De Boeck, G. Borghs. J. Appl. Phys. 66, 1739 (1989).

[17] M.P.C.M. Krijn. Semicond. Sci. Technol. 6, 27 (1991).

[18] Landolt-Börnstein. Band-22 Halbleiter, III/22a. SpringerVerlag, Berlin, Heidelberg (1987). V. 22. Ch. III. P. 22a.

[19] Л.П. Горьков, И.Е. Дзялошинский. ЖЭТФ 53, 717 (1967).

[20] А.В. Кавокин, А.И. Несвижский, Р.П. Сейсян. ФТП 27, 977 (1993).

[21] P. Bigenwald, B. Gil. Solid State Commun. 91, 33 (1994).

[22] A.V. Kavokin, A.I. Nesvizhskii. Phys. Rev. B 49, 17055 (1994).

[23] A.V. Kavokin, V.P. Kochereshko, G.R. Pozina, I.N. Uraltsev, D.R. Yakovlev, G. Landwehr, R.N. Bicknell-Tassius, A. Waag. Phys. Rev. B 46, 7713 (1992).

[24] Л.Г. Герчиков, А.В. Субашиев. ФТП 27, 446 (1993). 
[25] G.N. Aliev, N.V. Luk'yanova, R.P. Seisyan, M.R. Vladimirova, H. Gibbs, G. Khitrova. Phys. Status Solidi A 164, 193 (1997).

[26] Н.Д. Ильинская, С.И. Кохановский, Р.П. Сейсян. ФТП 27, 108 (1993).

[27] Г.Н. Алиев, Н.В. Лукьянова, Р.П. Сейсян. ФТТ 38, 1067 (1996).

[28] Р.П. Сейсян. Спектроскопия диамагнитных экситонов. М. (1984). $282 \mathrm{c}$.

[29] R.P. Seisyan, B.P. Zakharchenya. In: Landau level spectroscopy / Ed. G. Landwehr, E.I. Rashba. North-Holland, Amsterdam (1991). V. 1. P. 345 [Modern Problems in Condensed Matter Sciences. V. 27].

[30] G.N. Aliev, V.A. Kosobukin, N.V. Luk'yanova, M.M. Moiseeva, R.P. Seisyan, H. Gibbs, G. Khitrova. IOP Publishing Ltd. Inst. Phys. Conf. Ser. N 155 (1997). Ch. 2. P. 165.

[31] С.И. Кохановский, Ю.М. Макушенко, Р.П. Сейсян, Ал.Л. Эфрос, Т.В. Язева, М.А. Абдуллаев. ФТП 25. 493 (1991).

[32] А. Клочихин, А. Резницкий, Л. Тенишев, С. Пермогоров, С. Иванов, С. Сорокин, Х. Муманис, Р. Сейсян, С. Клинглирн. Письма в ЖЭТФ 71, 348 (2000).

[33] A.V. Kavokin. Phys. Rev. B 50, 8000 (1994).

[34] R.M. Datsiev, V.A. Kosobukin, N.V. Luk'yanova, R.P. Seisyan, M.R. Vladimirova. In: Excitonic Processes in Condensed Matter / Ed. R.T. Williams, W.M. Yen. The Electrochemical Society Proceedings Series. Pennington, N.Y. (1998). P. 98-25.

[35] N.J. Pulsford, R.J. Nicholas, R.J. Warburton, M.J. Lawless, G. Duggan, K.J. Moore, K. Woodbridge, C. Roberts. Superlat. Microstruct. 9, 521 (1991).

[36] A.V. Trifonov, S.N. Korotan, A.S. Kurdyubov, I.Ya. Gerlovin, I.V. Ignatiev, Yu.P. Efimov, S.A. Eliseev, V.V. Petrov, Yu.K. Dolgikh, V.V. Ovsyankin, A.V. Kavokin. Phys. Rev. B 91, 115307 (2015)

[37] H. Wang, H. Yu, X. Zhou, Q. Kan, L. Yuan, W. Chen, W. Wang, Y. Ding, J. Pan. Appl. Phys. Lett. 105, 141101 (2014).

[38] S. Gies, C. Kruska, C. Berger, P. Hens, C. Fuchs, A. Ruiz Perez, N.W. Rosemann, J. Veletas, S. Chatterjee, W. Stolz, S.W. Koch, J. Hader, J.V. Moloney, W. Heimbrodt. Appl. Phys. Lett. 107, 182104 (2015).

[39] R.P. Seisyan. Semicond. Sci. Technol. 27, 053001 (2012).

[40] Р.П Сейсян. ФТТ 58833 (2016). 